\title{
EVERYWHERE EQUIVALENT AND EVERYWHERE DIFFERENT KNOT DIAGRAMS*
}

\author{
ALEXANDER STOIMENOW ${ }^{\dagger}$
}

\begin{abstract}
A knot diagram is said to be everywhere different (resp. everywhere equivalent) if all the diagrams obtained by switching one crossing represent different (resp. the same) knot(s). We exhibit infinitely many everywhere different knot diagrams. We also present several constructions of everywhere equivalent knot diagrams, and prove that among certain classes these constructions are exhaustive. Finally, we consider a generalization to link diagrams, and discuss some relation to symmetry properties of planar graphs.
\end{abstract}

Key words. Alternating knot, semiadequate knot, Jones polynomial, Kauffman bracket, planar graph, edge transitive.

AMS subject classifications. Primary 57M25; Secondary 05C10, 57M15, 05C75.

1. Introduction. This paper is concerned with the study of two suggestive classes of knot (and partly link) diagrams, namely those for which the diagrams obtained by one crossing change all represent the same or all represent different knots.

A knot diagram is said to be everywhere different if all the diagrams obtained by switching one crossing represent different knots. This property was apparently first considered by K. Taniyama, who found a few everywhere different diagrams by computation. Taniyama (and independently Ishii for alternating diagrams) asked whether infinitely many such diagrams exist. He and Shinjo proposed a candidate series of examples.

In the first part of the paper $(\S 3)$, we will exhibit three infinite sequences of examples: two alternating ones, and the (non-alternating) one of Taniyama and Shinjo. We take two approaches, which rely on the Kauffman bracket [Kf] for the Jones polynomial in different ways. First (theorem 2) we apply the semiadequacy formulas in [St3, DL] in combination with the Menasco-Thistlethwaite Flyping theorem. This leads to a short and elegant proof, which, however, works only for a suitably chosen class of examples. We then introduce another method, which is more laborious, but can be applied rather generally. It is based on an algebraic formalism of the Temperley-Lieb category (see theorems 3 and 4 ).

The similar properties of everywhere non-trivial diagram and everywhere equivalent diagram are specified by demanding that all diagrams obtained by switching one crossing represent a non-trivial, resp. the same knot. These properties were also defined by Taniyama, and considered by Makoto Ozawa and Ryo Hanaki (unpublished). In [SA] we previously studied diagrams we should call (here) "everywhere trivial", which unknot at every crossing.

The second part of the paper $(\$ 4)$ is devoted to everywhere equivalent diagrams. First we consider the special case of everywhere trivial diagrams. We answer a question in [SA] regarding everywhere trivial unknot diagrams (proposition 2), using an idea in an example of everywhere non-trivial unknot diagrams by Shinjo and Taniyama. We then present several constructions of everywhere equivalent diagrams (proposition 3). We use the work in [St5, St6, SV] to show that among diagrams of low genus, only

\footnotetext{
*Received February 19, 2011; accepted for publication March 7, 2012.

$\dagger$ Department of Mathematics, Keimyung University, Dalseo-Gu, Sindang-Dong 1000, Daegu 704701, Korea (stoimeno@stoimenov.net).
} 
the expected ones are everywhere equivalent (theorem 5).

The discussion of everywhere equivalent diagrams finishes, in $\S 5$, with a longer discourse into some graph theory. We observe that our constructions are closely related to some well-studied (transitive) symmetry properties of planar graphs. We introduce then two graph-based methods for obtaining everywhere equivalent diagrams. Beside subsuming the preceding constructions in the case of knots, they give rise to new families of everywhere equivalent diagrams of links. With this different point of view on everywhere equivalence, it seems that the relationship between knot and graph theory is worth stressing in this context.

A large part of the paper bases on computations, performed with various tools, including KnotScape $[\mathrm{HT}]$, but most substantially using MATHEMATICA $^{\mathrm{TM}}$ [Wo]. Roughly the work here can be circumscribed by how to program MATHEMATICA to verify what is needed. Thus our attitude in the proofs will be mainly to explain the theoretical input into, and to guide through the calculation with the program.

The referee made a number of substantial comments on the paper, in response to which several portions were reorganized (and often expanded), with the hope to enhance readability and make the content more widely accessible. (Occasionally these changes are explicitly indicated.)

2. Preliminaries. It seems useful to collect various preliminaries, which will be used at different places throughout the proofs later in the paper.

2.1. Link diagrams. All link diagrams are considered oriented, even if orientation is sometimes ignored. We also assume here that we actually regard the plane in which a link diagram lives as $S^{2}$, that is, we consider as equivalent diagrams which differ by the choice of the point at infinity. By $! D$ we denote the mirror image of a diagram $D$, obtained by switching all crossings (as opposed to taking the planar mirror image).

A diagram $D$ is composite if there is a closed curve $\gamma$, called decomposing curve, transversally intersecting $D$ in two points outside the crossings, such that both the interior and the exterior of $\gamma$ contain crossings of $D$. If $D$ is not composite, then we call $D$ prime. A composite diagram $D$ can be decomposed along a decomposing curve $\gamma$ as a connected sum $D=D_{1} \# D_{2}$ of two diagrams $D_{1}$ and $D_{2}$. To obtain $D_{i}$, take the parts of $D$ in the interior and exterior of $\gamma$, and close each of the two with a trivial arc. This operation can be iterated until we have a prime (diagram) decomposition $D=\#_{i} D_{i}$ of $D$. It will often be enough to look at prime diagrams $D$, and we will assume our diagrams to be so, unless we speak explicitly of composite ones.

The writhe, or (skein) sign, is a number, +1 or -1 , assigned to every crossing in a link diagram. A crossing as on the left in (1) has writhe 1 and is called positive. A crossing as in the middle of (1) has writhe -1 and is called negative. The writhe $w(D)$ of a link diagram $D$ is the sum of writhes of all its crossings. A diagram is called positive (see e.g. [St, O]) resp. negative, if all its crossings are positive resp. negative.

2.2. Jones polynomial. The Jones polynomial $V$ can be defined as the polynomial taking the value 1 on the unknot, and satisfying the skein relation

$$
t^{-1} V(\nwarrow)-t V(\nearrow)=\left(t^{1 / 2}-t^{-1 / 2}\right) V(\nearrow \circlearrowright)
$$

We will denote in each triple as in (1) the link diagrams (from left to right) by $D_{+}$, $D_{-}$and $D_{0}$; they are understood to be identical except at the designated spot.

It is useful to give here the alternative description of $V$ via Kauffman's state model [Kf]. A state is a choice of splicings (or splittings) of type $A$ or $B$ (see figure 
1) for any single crossing of a link diagram $D$. We call the $A$-state $A(D)$ the state in which all crossings are $A$-spliced, and the $B$-state $B(D)$ is defined analogously.

When for a state $S$ all splicings are performed, we obtain a splicing diagram, which consists of a collection of (disjoint) circles in the plane (solid lines) together with crossing traces (dashed lines). This splicing diagram is called in combinatorics $a$ perfect matching in a planar trivalent graph. We will for convenience identify below a state $S$ with its splicing diagram for fixed $D$. We will thus talk of the loops and traces of a state.

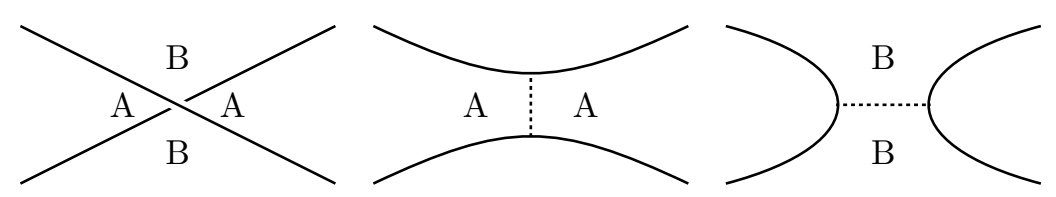

FIG. 1. The A- and B-corners of a crossing, and its both splittings. The corner A (respectively $B$ ) is the one passed by the overcrossing strand when rotated counterclockwise (respectively clockwise) towards the undercrossing strand. A type A (resp. B) splitting is obtained by connecting the $A$ (resp. $B)$ corners of the crossing. The dashed line indicates the trace of the crossing after the split.

Recall, that the Kauffman bracket $\langle D\rangle[\mathrm{Kf}]$ of a link diagram $D$ is a Laurent polynomial in a variable $A$, obtained by a sum over all states $S$ of $D$ :

$$
\langle D\rangle=\sum_{S} A^{\# A(S)-\# B(S)}\left(-A^{2}-A^{-2}\right)^{|S|-1},
$$

where \# $A(S)$ and \#B(S) denote the number of type A (respectively, type B) splittings and $|S|$ the number of (solid line) loops in the splicing diagram of $S$. The formula (2) results from applying the first of the bracket relations

$$
\langle\rangle\rangle=A^{-1}\langle\asymp\rangle+A\langle)(\rangle,\langle\bigcirc \cup X\rangle=\left(-A^{2}-A^{-2}\right)\langle X\rangle,
$$

to each crossing of $D$, and then deleting (except one) isolated loops using the second relation, at the cost of a factor $-A^{2}-A^{-2}$. (The normalization is thus here that one loop with no crossings has unit bracket.)

The Jones polynomial of a link $L$ can be determined from the Kauffman bracket of some diagram $D$ of $L$ by

$$
V_{L}(t)=\left.\left(-t^{-3 / 4}\right)^{-w(D)}\langle D\rangle\right|_{A=t^{-1 / 4}},
$$

with $w(D)$ being the writhe of $D$. This is another way, different from (1), to specify the Jones polynomial.

It is well-known that $V \in \mathbb{Z}\left[t^{ \pm 1}\right]$ (i.e., only integral powers occur) for odd number of link components (in particular, for knots), while $V \in t^{1 / 2} \cdot \mathbb{Z}\left[t^{ \pm 1}\right]$ (i.e., only halfintegral powers occur) for even number of components.

2.3. Semiadequacy and adequacy. Let $S$ be the $A$-state of a diagram $D$ and $S^{\prime}$ a state of $D$ with exactly one $B$-splicing. If $|S|>\left|S^{\prime}\right|$ for all such $S^{\prime}$, we say that $D$ is $A$-adequate. Similarly one defines a $B$-adequate diagram $D$. See [LT]. Then we 
set a diagram to be

$$
\begin{aligned}
\text { adequate } & =\mathrm{A} \text {-adequate and } \mathrm{B} \text {-adequate, } \\
\text { semiadequate } & =\mathrm{A} \text {-adequate or } \mathrm{B} \text {-adequate, } \\
\text { inadequate } & =\text { neither } \mathrm{A} \text {-adequate nor } \mathrm{B} \text {-adequate. }
\end{aligned}
$$

(Note that inadequate is a stronger condition than not to be adequate.)

A link is called $A$ (or $B$ )-adequate, if it has an $A$ (or $B$ )-adequate diagram. It is semiadequate if it is $A$ - or $B$-adequate, and inadequate, if it is not semiadequate, that is, neither $A$ - nor $B$-adequate. A link is adequate if it has an adequate diagram. This property is stronger than being both $A$ - and $B$-adequate, since a link might have diagrams that enjoy either properties, but none that does so simultaneously. The Perko knot $10_{161}$ in [Ro, appendix] is an example. This property of the Perko knot follows from work of Thistlethwaite [Th2], and is explained, e.g., in Cromwell's book [Cr, p. 234], or (along with further examples) in [St9].

It is easily observed (as in $[\mathrm{LT}]$ ) that a reduced alternating diagram (and hence an alternating link) is adequate.

For $V \in \mathbb{Z}\left[t^{1 / 2}, t^{-1 / 2}\right]$, the minimal or maximal degree min $\operatorname{deg} V$ or $\max \operatorname{deg} V$ is the minimal resp. maximal exponent of $t$ with non-zero coefficient in $V$. Let $\operatorname{span} V=$ $\max \operatorname{deg} V-\min \operatorname{deg} V$. The coefficient in degree $d$ of $t$ in $V$ is denoted $[V]_{t^{d}}$ or $[V]_{d}$. More often, however, we will need to designate coefficients in degrees relative to the minimal and maximal degree of the polynomial. In that case, we will use the below notation.

Let $V \in \mathbb{Z}\left[t^{ \pm 1 / 2}\right]$ have only integral or only half-integral powers, and $n \geq 0$ be an integer. Let $m=\min \operatorname{deg} V$ and $M=\max \operatorname{deg} V$ (then $2 m, 2 M \in \mathbb{Z}$ ). We write $V_{n+1}(V):=[V]_{m+n}$ and $V_{-n-1}(V):=[V]_{M-n}$ for the $n+1$-st or $n+1$-last coefficient of $V$. Similarly we set $V_{n}(D):=V_{n}(V(D))$ and $V_{n}(L):=V_{n}(V(L))$ for a link $L$ or a diagram $D$.

A basic observation in [LT] is that when $L$ is $A$ - resp. $B$-adequate then $\left|V_{1}(L)\right|=$ 1 resp. $\left|V_{-1}(L)\right|=1$. Thus if $L$ is adequate, and in particular alternating, both properties hold.

When $L$ is an $A$-adequate link, then $V_{n}(L)$ for $n \leq 3$ were studied in [DL, St3]. We will need the formulas below, without giving the details of their application, but for completeness the formulas should be explained. Let $D$ be an $A$-adequate diagram of $L$. (We will assume that $D$ is connected.) Then, in the notation of [St3],

$$
\left|V_{2}(D)\right|=e-|A(D)|+1
$$

and

$$
\left|V_{3}(D)\right|=\left(\begin{array}{c}
\left|V_{2}(D)\right|+1 \\
2
\end{array}\right)+e_{++}+\delta-\triangle
$$

Here $|A(D)|$ is the number of loops in the $A$-state $A(D)$, and the quantities $e, e_{++}$, $\delta$, and $\triangle$ are the number of pairs or triples of loops in $A(D)$ for which there exist crossing traces (obtained as in figure 1) making them look (up to moves in $S^{2}$ ) like 
in:

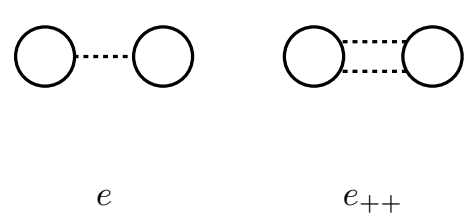

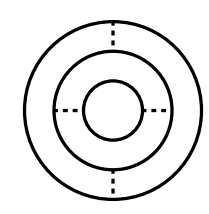

$\delta$

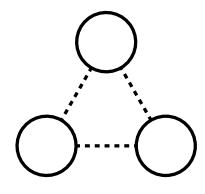

$\triangle$

(We do not require that these be the only traces connecting the loops, only that such traces should exist.) Note that when $D$ is alternating, then $\delta=0$.

2.4. Graphs. Let in the following a graph be finite, i.e., with finitely many edges and vertices, and planar, i.e., having a fixed planar embedding (this is often called a planar map). Let us, again, for convenience assume that we actually regard the plane as $S^{2}$, that is, we take the freedom to move the point at infinity.

A graph may have several edges connecting the same two vertices. For two such vertices, the $n$ edges connecting them (when $n>1$ ) are called multiple edges, and are not regarded as one edge (of multiplicity $n$ ). Thus a multiple (resp. double or triple) edge is an edge with some (resp. exactly 1 or 2 ) other edge(s) connecting the same two vertices. We write $E(G)$ for the set of edges of $G$.

A planar graph $G$ has a planar dual $G^{*}$. We will assume $G$ to be 2 -connected (no isthmuses) and dually 2-connected (no loop edges).

Let $M_{q}$ be the graph of two vertices with $q$ edges. This is the planar dual of the cycle of length $q$. In particular, $M_{1}$ is a single edge, $M_{2}$ is a 2-cycle, and $M_{3}$ is the graph often called theta-curve.

For a graph, let the operation

(adding a vertex of valence 2) be called bisecting. The doubling of an edge consists in adding a new edge connecting the same two vertices in a way that a new 2 -gonal region is created in the plane. The doubled graph $G$ is the graph obtained from $G$ by doubling all its edges.

Recall the checkerboard graph construction. It associates a connected graph $G=$ $G(D)$ to an unoriented connected link diagram $D$ (and vice versa), in the following manner. Consider the plane curve $\hat{D}$ of $D$ as a 4 -valent planar graph. There is a black-white coloring of the regions of $\hat{D}$, the checkerboard coloring, which assigns different colors to each pair of regions opposite at some edge of $\hat{D}$. Then $G(D)$ is defined to have a vertex for each black region of $\hat{D}$ and an edge for each crossing. See figure 2 (or also, e.g., [Th, Fig. 5] or [St4, MS, Kf]). We should note that $G(D)$ is in fact a planar graph, i.e., comes with a planar embedding.

When a checkerboard graph $G$ of $D$ is fixed, each crossing of $D$ carries a Kauffman sign. Let a crossing $c$ of $D$, or its corresponding edge $e$ in $G$, be Kauffman positive (resp. Kauffman negative) if the $A$-corners (resp. $B$-corners) of $c$ in figure 1 come from 

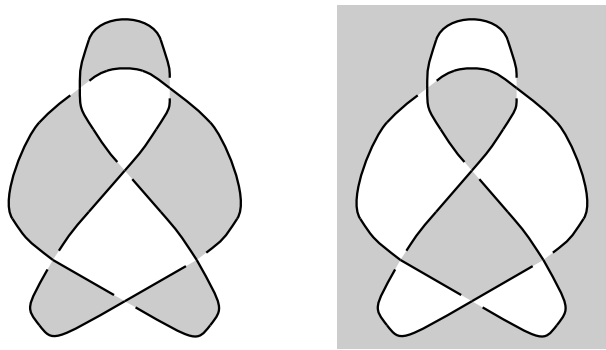

two checkerboard colorings

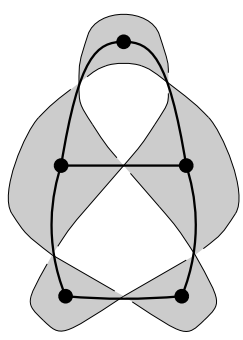

the checkerboard graph of the first coloring

FIG. 2. Checkerboard coloring and checkerboard graph.

the black regions of the checkerboard coloring $G$ was obtained from ${ }^{1}$.

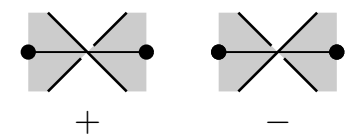

Since Kauffman signs are an unoriented feature, they are not the same as the (skein) signs from (1); there is, though, a relationship between both, which will occur later. Since the separation between skein and Kauffman signs of crossings is fundamental, we will still often write "(skein) sign" instead of simply "sign".

Observe that a diagram is alternating iff all its crossings have the same Kauffman sign. For example, it is always negative in the right diagram of figure 2 .

The median graph (or sometimes also called line graph) $M(G)$ of $G$ is a planar 4-valent graph obtained as follows: vertices of $M(G)$ are edges of $G$, and an edge connects $v_{1}$ and $v_{2}$ in $M(G)$ if and only if $v_{1}$ and $v_{2}$ are edges incident to the same vertex in $G$ and in the boundary of the same face of $G$.

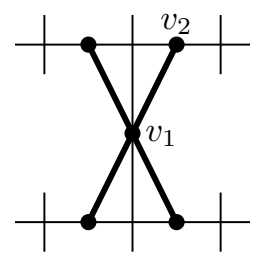

(Here the thick lines correspond to edges in $M(G)$ and the thin ones to edges in $G$.) To conform to 4 -valence, if a bigonal region is formed by edges $v_{1}$ and $v_{2}$ in $G$, two edges should connect vertices $v_{1}$ and $v_{2}$ in $M(G)$.

The plane curve of $D$, regarded as a 4 -valent graph, is the median graph of $G(D)$. Thus, given $G=G(D)$, one can reconstruct $D$ when Kauffman signs of the crossings are specified. In particular, an alternating diagram $D$ is determined up to mirror image.

\footnotetext{
${ }^{1}$ Both the choice of checkerboard color $G=G(D)$ is built from, and the choice of Kauffman sign, are widely unstandardized conventions: they are sometimes followed, and sometimes reverted in other articles as compared to here.
} 
Note that $M\left(G^{*}\right)=M(G)$, and thus graphs dual to each other give rise to the same alternating diagram $D$ (up to mirror image). For non-alternating diagrams, the Kauffman signs of all crossings change when $G$ is replaced by its dual. On the other hand, $D$ determines $G$ up to duality. In this sense, we will below speak of the graph of a diagram and the diagram of a graph.

The star $\operatorname{star} v$ of a vertex $v$ in $G$ is the set of incident edges. A cut vertex is a vertex $v$ in $G$ for which deletion of $v$ along with all edges in star $v$ (but keeping their adjacent vertices different from $v$ ) disconnects $G$. A graph $G$ is cut-free, if it has no cut vertex. A connected link diagram $D$ is prime (as defined in $\S 2.1$ ) if and only if $G(D)$ is cut-free.

A symmetry of the graph is for us a permutation of its vertices and edges induced by a homeomorphism of the plane. Such a homeomorphism is a deformation of the identity or of the inversion of the plane. A graph is edge transitive if for every two edges $e, e^{\prime}$ there is a symmetry mapping $e$ to $e^{\prime}$.

2.5. Conway/Alexander polynomial and Determinant. The Conway polynomial $\nabla(z)$ is given by its value 1 on the unknot and a property similar to (1):

$$
\nabla(\nearrow)-\nabla(\nearrow)=z \nabla() \circlearrowright)
$$

This polynomial is an equivalent of the Alexander polynomial $\Delta(t)$ :

$$
\Delta(t)=\nabla\left(t^{1 / 2}-t^{-1 / 2}\right) .
$$

(For the shape of $\nabla(z)$, see $\S 2.6$.)

By putting $t=-1$ into the skein relations for $V$ and $\Delta$, one sees easily that $V(-1)= \pm \Delta(-1)$, the sign depending on the parity of ne of components of the link. Thus $V$ and $\Delta$ contain the common invariant

$$
\operatorname{det}(L):=|\Delta(L)(-1)|=|V(L)(-1)|,
$$

which is called determinant of $L$. The reasons for this name and the significance of this quantity should not be discussed here. We will need, however, some well-known properties of the determinant.

Lemma 1. Let $D$ be an alternating diagram. Then $\operatorname{det}(D)$ is equal to the number of spanning trees of a checkerboard graph $G(D)$ of $D$.

Proof. There are several explanations of this fact. One comes from the Kauffman bracket.

Notice that when $t^{ \pm 1}=A^{\mp 4}=-1$, then the sum in (2) collapses to the sum over the, so-called monocyclic, states $S$, those with one loop. Such states correspond one-to-one to spanning trees of a fixed checkerboard graph of $D$. (Of course, the number of spanning trees is invariant under duality.) For a monocyclic state, look at the subgraph of $G$ which is inside one of the regions of the loop. For a spanning tree $\Gamma$, splice all crossings corresponding to edges of $\Gamma$ so that the black regions (of the checkerboard coloring giving rise to $G$ ) are joined.

Since in an alternating diagram all crossings have the same Kauffman sign, in the expression $(2)$ of $\pm V(D)(-1)$ as the sum of \pm 1 over the spanning trees of the checkerboard graph, all terms have the same sign.

For more details see, e.g., [St4]. There is also a similar explanation using the Alexander polynomial, which can be found, e.g., in [MS]. 
LEMma 2. Let $D$ be a prime alternating diagram and $D^{\prime}$ be a non-alternating diagram obtained from $D$ by applying crossing changes (i.e., with the same plane curve). Then $\operatorname{det}\left(D^{\prime}\right)<\operatorname{det}(D)$.

Proof. This follows rather directly from the previous proof. If $G=G\left(D^{\prime}\right)$ has no cut vertex, and edges of opposite Kauffman sign, then for two different spanning trees of $G$ the terms \pm 1 in (2) will have opposite sign, and will cancel.

2.6. 2-divisibility of the determinant. There is a 2-divisibility property of the determinant, which seems not often mentioned, but easily results, e.g., from the description of values taken by the Conway/Alexander polynomial. It is well-known, that for a knot $K$, the Conway polynomial is of the form $\nabla(K)=1+z^{2} P\left(z^{2}\right)$ for some $P \in \mathbb{Z}[z]$, while for an $n$-component link $L$, we have $\nabla(L)=z^{n-1} \cdot P\left(z^{2}\right)$. By noticing that

$$
\Delta(-1)=\nabla(2 \sqrt{-1})
$$

we immediately see the following:

Proposition 1. A link $L$ had odd determinant if and only if $L$ is a knot. When $L$ has $n$ components, then $2^{n-1} \mid \operatorname{det}(L)$.

As a consequence, the following relationship was observed essentially in [St4, MS]:

LemMa 3. A link diagram $D$ represents a knot if and only if its checkerboard graph $G=G(D)$ has an odd number of spanning trees. For an n-component link diagram $D$, this number is divisible by $2^{n-1}$.

Proof. Number of components is not changed by choosing $D$ alternating. For such $D$, the number of spanning trees of $G$ is equal to the determinant by lemma 1 . Then apply proposition 1 .

Note that this lemma has an entirely combinatorial reformulation: it relates the 2-divisibility of the number of spanning trees of a planar graph $G$ to the number of (transversely intersecting) planar loops that form $M(G)$. When $G$ is additionally bipartite, many more properties were given in [MS]. It seems legitimate to ask whether there is a self-contained framework to explain these relations independently from knot theory.

2.7. Vassiliev invariants. The degree- 2 Vassiliev knot invariant $v_{2}$ is given by the coefficient of $z^{2}$ in $\nabla(z)$. This invariant can be also expressed from the Jones polynomial as $v_{2}=-1 / 6 V^{\prime \prime}(1)$. The normalization of the degree- 3 invariant $v_{3}$ (see e.g. $[\mathrm{PV}, \mathrm{St}])$ we will use is

$$
v_{3}=-\frac{1}{12} V^{\prime \prime}(1)-\frac{1}{36} V^{\prime \prime \prime}(1)
$$

It is worth remarking (and we will use below) that $v_{2}$ and $v_{3}$ enjoy a maximizing property for positive diagrams similar to the one of the determinant for alternating diagrams in lemma 2.

Lemma 4. (see [St, Theorem 5.2 and Exercise 6.2]) Let $D$ be a positive knot diagram and $D^{\prime}$ be a non-positive diagram obtained from $D$ by applying crossing changes (at least one). Then $v_{2}\left(D^{\prime}\right)<v_{2}(D)$ (unless $D^{\prime}=! D$ ) and $v_{3}\left(D^{\prime}\right)<v_{3}(D)$. 
We will also need a few times the degree- 4 invariant $v_{4}$ being the $z^{4}$-coefficient in $\nabla(z)$, and once the degree- 5 invariant given by

$$
v_{5}=\frac{1}{120} V^{(5)}(1)
$$

(where the parenthesized superscript means derivative).

Note more generally, that the invariants $V^{(n)}(1)$ are Vassiliev invariants of degree $n$, and that these determine $V($ see $[\mathrm{BN}])$.

2.8. Flypes. Next, we briefly clarify the use of flypes and the MenascoThistlethwaite theorem.

Definition 1. A flype is a move on a diagram shown in figure 3. Note that if one of the tangles $P$ or $Q$ contains only (possibly zero) horizontal twists, the flype is trivial (does not alter the diagram). We say that a diagram admits a flype if it can be represented as one of the diagrams in the figure, and neither $P$ nor $Q$ contain only horizontal twists.

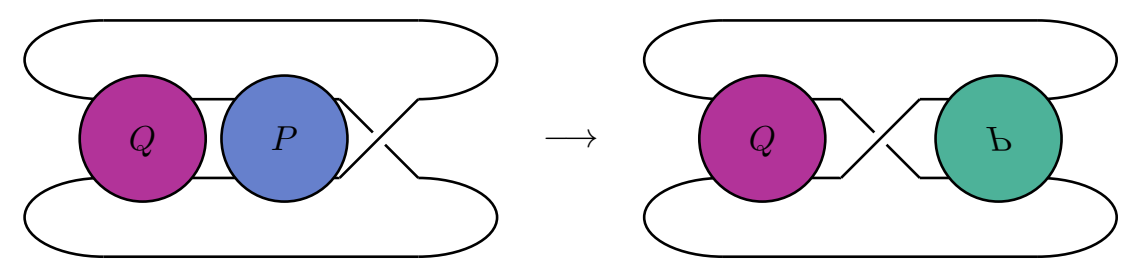

FIG. 3. A flype near the crossing $p$.

By the fundamental work of Menasco-Thistlethwaite, we have a proof of the Tait flyping conjecture.

THEOREM 1. ([MT]) For two alternating diagrams of the same alternating link, there is a sequence of flypes (and moves in $S^{2}$ ) taking the one diagram into the other.

2.9. Genus and Generators. We summarize very briefly the tools for the treatment of the diagram genus developed in [St5, St6, SV].

We first recall the genus of a knot diagram. The replacement of a (positive or negative) crossing by the the rightmost picture in (1) is called smoothing out. When all crossings of $D$ are smoothed out, we have a collection of loops called Seifert circles. Let $s(D)$ be the number of Seifert circles of $D$ and $c(D)$ the number of its crossings. We recall that the genus of a knot diagram $D$ is given by

$$
\frac{1}{2}(c(D)-s(D)+1) .
$$

Two crossings $p$ and $q$ in a knot diagram $D$ are linked if the cyclic order of the basepoints is $p q p q$ (and not $p p q q$ ), or equivalently, if smoothing out both crossings gives a knot (and not a 3-component link) diagram. Another way of saying this is: for a given crossing $p$ of $D$, a crossing $q$ is linked with $p$ iff $q$ involves strands of two different components, i.e., is a mixed crossing, of the (2-component) link diagram obtained by smoothing out $p$ in $D$. Notice in a link diagram the number of mixed 
crossings is even, and thus so is the number of crossings $q$ linked with a given crossing $p$ in a knot diagram. We called this in [St] the even valence property.

We call two crossings $p$ and $q$ to be $\sim$-equivalent if $p$ and $q$ form a reverse clasp up to flypes. For instance, in the below fragment, $q$ and $r$ form a reverse clasp, thus they are $\sim$-equivalent. However, after the tangle $Q$ is flyped to the right (and $p$ moves to the left), $p$ and $q$ form a reverse clasp, too. Thus also $p$ and $q$ are $\sim$-equivalent.

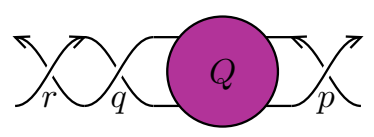

For a knot diagram, -equivalence of crossings $p$ and $q$ can be reformulated by saying that $p$ and $q$ are not linked, but each $r \neq p, q$ is linked with $p$ iff $r$ is linked with $q$. (In this description, one easily sees $\sim$ to be an equivalence relation from the even valence property.)

A knot diagram decomposes into -equivalence classes, which we often simply call 'classes' below. We can then talk of (un)linked classes $S, T$, when some (or any) crossing in $S$ is (un)linked with some (or any) crossing in $T$.

$\mathrm{A} \bar{t}_{2}^{\prime}$ move is one replacing a crossing by three $\sim$-equivalent ones of the same sign. Up to mirroring this is given by

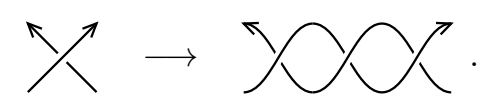

(For example, the 5 crossings in the reverse twist tangle of (61) can be obtained from one crossing by applying two $\bar{t}_{2}^{\prime}$ moves.) A reverse $\bar{t}_{2}^{\prime}$ move acts in the opposite direction, and reduces three $\sim$-equivalent crossings to one. In certain cases, a diagram admits no reverse $\bar{t}_{2}^{\prime}$ move, but does so after a flype. We saw such an example in (13), where crossing $p$ can be moved close to crossing $q$ by flyping the tangle $Q$, and then a reverse $\bar{t}_{2}^{\prime}$ move can be applied on $p, q$ and $r$ (to discard two of them).

An diagram is called a generator if it is alternating and if each $\sim$-equivalence class of its crossings has 1 or 2 elements. That is, generator diagrams are the alternating diagrams which cannot be reduced further by reverse $\bar{t}_{2}^{\prime}$ moves after any sequence of flypes.

The set of diagrams obtained from a generator $\hat{D}$ by flypes, crossing changes, and $\bar{t}_{2}^{\prime}$ moves is called the series of $\hat{D}$.

By abuse of terminology, we call a generator also a knot (or link) with a generating diagram, or equivalently, we consider generator diagrams up to flypes. Of course, by theorem 1, if one diagram is a generator, so are all diagrams of the same knot (or link). Working with one diagram per generator knot instead of all diagrams is justified as long as the statements asserted and methods employed in the proof are invariant under (diagram) mutations.

The connected sum of two generators is again a generator (of the sum of the genera). These can often be disregarded, and thus it is relevant to consider only prime generators.

A fundamental feature of generator knots ([St5]) is that there are only finitely many of given genus. Their list has been obtained up to genus 4 in [St6, St8], and forms a basis of our later inspective efforts. We cannot repeat here details of the 
generation of these lists, but we summarize their size in the below table.

\begin{tabular}{c||c|c|c|c} 
genus & 1 & 2 & 3 & 4 \\
\hline number of prime generators & 2 & 24 & 4,017 & $3,414,819$
\end{tabular}

A clasp is the tangle with two crossings. A clasp is trivial or resolved if both crossings have opposite sign (i.e., can be canceled by a Reidemeister II move).

If a diagram $D$ has no trivial clasp up to flypes, then $D$ has no class that contains crossings of either sign. We will refer to such classes below as unsigned; otherwise the class is signed, and positive resp. negative depending on the (skein) sign of its crossings. Diagrams with no unsigned class of more than 2 crossings admit a parametrization by a twist vector, which we will work with, and thus which describe now.

Consider a generator $\hat{D}$ with $g$ classes $^{2}$. A diagram in the series of $\hat{D}$ is given by $\hat{D}_{y}$ for a twist vector $y=\left(y_{i}\right)_{i=1}^{g}$, where $y_{i}$ are integers. This diagram $\hat{D}_{y}$ is defined by saying that class $i$ in $\hat{D}_{y}$ should have writhe

$$
w_{i}=2 y_{i}-2+\# i,
$$

where $\# i \in\{1,2\}$ is the number of crossings in $\sim$-equivalence class $i$ of $\hat{D}$. Let us call a class $i$ even or odd depending on \#i. The writhe $w_{i}$ is understood to be realized by $\left|w_{i}\right|$ crossings of skein $\operatorname{sign} \operatorname{sgn}\left(w_{i}\right)$, except for $w_{i}=0$, where we consider a resolved clasp (one positive and one negative crossing).

\section{Examples of everywhere different diagrams.}

3.1. An example based on semiadequacy. We consider an alternating diagram $D_{n}$ of $8+2 n$ crossings formed from the tangle $T$ by composing it with an oriented braid tangle $\left(\sigma_{1} \sigma_{2}^{-1}\right)^{n}$ and closing up. The composition for $n=7$ (prior to closing up and moving two $\sigma_{1} \sigma_{2}^{-1}$ on the other side) is shown on the right of figure 4 .
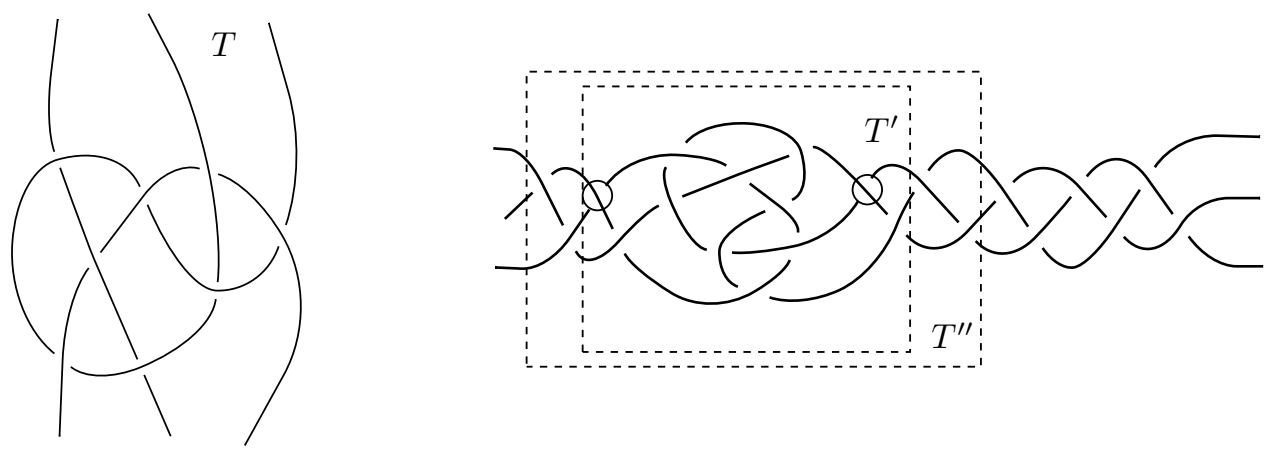

FIG. 4.

TheOREm 2. For almost all $n=3 k+1$, the diagram $D_{n}$ is everywhere different.

Proof. It will be helpful to introduce also the tangles $T^{\prime}$ and $T^{\prime \prime}$ obtained from $T$ by incorporating the outermost 1 resp. 2 powers of $\sigma_{1} \sigma_{2}^{-1}$ in the braid tangle.

\footnotetext{
${ }^{2}$ Unlike here, in the cited papers, $g$ stands for the genus.
} 
Number the crossings inside $T^{\prime \prime}($ not $T$ ) by $1, \ldots, 16$. It is not very important which crossing receives which number, however, we will need some particular attention to the two encircled crossings, which in our numbering received labels 2 and 6 (see below).

Let $D_{n, i}$ for $i=1, \ldots, 16$ be $D_{n}$ with the crossing labeled as $i$ in $T^{\prime \prime}$ turned around, and $K_{n, i}$ the knot $D_{n, i}$ represents. Similarly let $D_{n, i, j}$ and $K_{n, i, j}$ be $D_{n}$ with the $j$-th crossing in the $i$-th power of $\sigma_{1} \sigma_{2}^{-1}$ in the braid tangle turned around. (A few diagrams then receive two names.)

First, the knot $K_{n}$ of $D_{n}$ is different from $K_{n, i}$ and $K_{n, i, j}$ because of the determinant (lemma 2).

We took $n=7$ and calculated $V\left(K_{n, i}\right)$ and $V\left(K_{n, i, j}\right)$. All they were distinct. In the following we explain how to infer from the case $n=7$ to the general case.

The $K_{n, i, j}$ for $1<i<n$ simplify to an alternating diagram of $4+2 n$ crossings, which can be distinguished by theorem 1. (One has to observe that these diagrams are mutually distinct, even up to moves in the 2-sphere, and none admits flypes.)

The $K_{n, i}$ can be distinguished as follows. Since $V\left(K_{7, i}\right)$ are distinct for all $i$, for each $1 \leq i<j \leq 16$, there is a Vassiliev invariant $v=v_{i, j}$ (obtained from $V$ as discussed at the end of $\S 2.7)$ distinguishing $K_{7, i}$ and $K_{7, j}$. Now $K_{3 k+1, i}$ and $K_{3 k+1, j}$ form braiding sequences, so the map $k \mapsto v\left(K_{3 k+1, i}\right)$ is a polynomial in $k$ [St2]. Polynomials differing at one spot differ almost everywhere, and thus, excluding finitely many $k$, we have that $v$ distinguishes $K_{3 k+1, i}$ and $K_{3 k+1, j}$. By applying this argument on $v_{i, j}$ for all $i, j$, we are done.

The most interesting case is how to distinguish $K_{n, m}$ from $K_{n, i, j}$ when $2<i<$ $n-1$. If the crossing $m$ does not belong to $T^{\prime}$, we distinguished them by theorem 1 . So we assume the switched $m$-th crossing of $T^{\prime \prime}$ belongs to $T^{\prime}$.

For this we use the semiadequacy formulas in [St3]; see $\S 2$. Let $V_{ \pm p}$, as in $\S 2$, be the $p$-th resp. $p$-th last coefficient of $V$ (where powers of $t$ are ordered increasingly).

It is easy to see from the alternating $4+2 n$ crossing diagrams and the semiadequacy formulas (6) and (7) that when $2<i<n-1, j=1,2$, then all $K_{n, i, j}$ have the same $V_{p}$ for $|p| \leq 3$. We denote by $\tilde{V}_{p}$ these values when $n$ is fixed.

Moreover, one can check that except for two $m$, the $D_{n, m}$ either

- simplify to a diagram of $<4+2 n$ crossings, or to a non-alternating diagram of $4+2 n$ crossings, or

- simplify through an isotopy of $T^{\prime}$ to a semiadequate diagram $D_{n, m}^{\prime}$.

We check next for $n=7$ that when $D_{n, m}^{\prime}$ is $A$-adequate, not all $\left(\operatorname{sgn}\left(V_{1}\right)\right.$. $\left.V_{p}\right)\left(D_{n, m}^{\prime}\right)$ for $1 \leq p \leq 3$ match $\operatorname{sgn}\left(\tilde{V}_{1}\right) \cdot \tilde{V}_{p}$. Similarly we check that when $D_{n, m}^{\prime}$ is $B$-adequate, then $\left(\operatorname{sgn}\left(V_{-1}\right) \cdot V_{-p}\right)\left(D_{n, m}^{\prime}\right)$ for some $1 \leq p \leq 3$ does not match $\operatorname{sgn}\left(\tilde{V}_{-1}\right) \cdot \tilde{V}_{-p}$.

Now the change from $n \geq 7$ to $n+3$ affects the quantities (8) that determine $V_{p}$ in a prescribed way. (The details can be easily worked out, and we omit them. Let us notice, however, that here it is important that the isotopy simplifying $D_{n, m}$ to $D_{n, m}^{\prime}$ acts inside $T^{\prime}$.) Thus it is easy to see that when they do not match for $n=7$, they will not either for general $n$.

There are two crossings in $T^{\prime}$ whose switch does not simplify to a semiadequate diagram; they are encircled in figure 4 . Let the right crossing have $m=6$ and the left $m=2$.

Both crossings are negative. We can obtain $V\left(D_{n, m}\right)=V\left(D_{n, m}^{\prime}\right)$ from $V\left(D_{n}\right)$ 
and $V$ of another alternating link $\tilde{D}_{n, m}$ via the skein relation (1):

$$
V\left(D_{n, m}\right)=\left(t^{3 / 2}-t^{-1 / 2}\right) V\left(\tilde{D}_{n, m}\right)+t^{2} V\left(D_{n}\right)
$$

Note that since $D_{n}$ and $\tilde{D}_{n, m}$ are alternating, we can control the 3 outermost terms of either summands on the right by the semiadequacy formulas (6) and (7). The formulas imply that for $n \geq 4$ and fixed $m$ both $V_{p}\left(D_{n}\right)$ and $V_{p}\left(\tilde{D}_{n, m}\right)$ are (up to sign)

- 1 for $p= \pm 1$,

- linear in $n$ with linear coefficient 1 for $p= \pm 2$ (for both $D_{n}$ and $\tilde{D}_{n, m}$, but with possibly different absolute term between $D_{n}$ and $\left.\tilde{D}_{n, m}\right)$, and

- quadratic in $n$ with quadratic coefficient $1 / 2$ (and possibly different lower degree terms) for $p= \pm 3$.

In particular, the values $V_{p}\left(D_{n}\right)$ and $V_{p}\left(\tilde{D}_{n, m}\right)$ in dependence on $n$ can be determined from two values of $n \geq 4$.

It remains to look what cancellations occur. In the case $n=7$ we have

- $\min \operatorname{deg} V\left(D_{n}\right)=-14$ and $\max \operatorname{deg} V\left(D_{n}\right)=8$,

- $\min \operatorname{deg} V\left(D_{n, 2}\right)=-10$ and $\max \operatorname{deg} V\left(D_{n, 2}\right)=7$,

- $\min \operatorname{deg} V\left(D_{n, 6}\right)=-10$, with $V_{1}\left(D_{n, 6}\right)=-2\left(\right.$ and $\left.\max \operatorname{deg} V\left(D_{n, 6}\right)=8\right)$.

For $m=2$ we see that when $n=7$, the two lowest and three highest terms cancel on the right of (16). By checking this also for $n=4$, we conclude that this occurs for general $n$, and so span $\left(V\left(D_{n, 2}\right)\right) \leq 3+2 n$, which cannot be the Jones polynomial of an alternating $4+2 n$ crossing knot.

For $m=6$ we see from (16), that for $n=7$ the two lowest terms cancel, and the third lowest term gives $V_{1}\left(D_{n, 6}\right)=-2$, which does not occur for the Jones polynomial of any alternating knot. By checking the same also for $n=4$, we have the general case.

With this the proof is finished.

The examples were clearly chosen here so as to have a simple proof. For other examples the proof appears considerably more complicated. We now introduce a method which accomplishes this. It is more laborious, but much more generally applicable.

3.2. An example based on the Temperley-Lieb category. We consider an example studied by Shinjo and Taniyama.

$T$

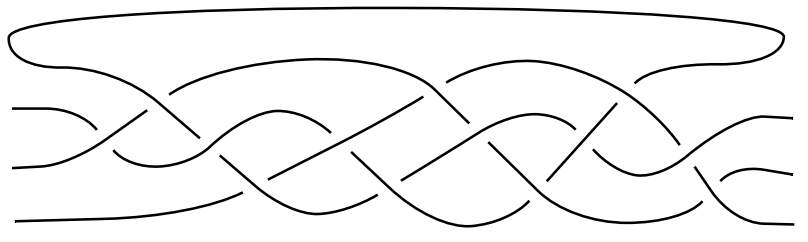

FIG. 5.

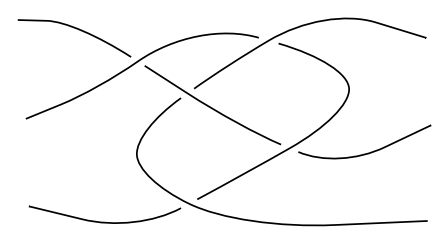

Let $D_{n}$ be the (alternating) knot diagram obtained by composing $T$ in figure 5 with $n$ copies of $T^{\prime}$ and closing up. Shinjo and Taniyama had verified that $D_{1}$ is everywhere different.

THEOREM 3. For almost all $n$, the diagram $D_{n}$ is everywhere different. 
Proof. Let $D_{n, i, j}$ be the diagram obtained by switching the $j$-th crossing in the $i$-th tangle $T^{\prime}$ (or $T$ if $i=0$ ). The number of crossings in $T$ and $T^{\prime}$ will be fixed in a moment.

We will not make any use whatsoever of alternation of $D_{n}$, and distinguish $D_{n, i, j}$ by a value of the Kauffman bracket. The variable $A$ in the bracket will be understood as a complex number, and will in fact use only two values,

$$
A_{0}=7 / 8-\sqrt{-1} / 8 \text { and } A_{0}^{\prime}=1-\sqrt{-1} / 8 .
$$

Their choice will be justified shortly. (A handful of other values served for additional checks, but it is not necessary to discuss these here.)

We used MATHEMATICA ${ }^{\mathrm{TM}}[\mathrm{Wo}$ for our calculations. It allows us to work exactly and numerically. Both modi are important. In certain cases the coincidence or vanishing of certain terms decisively impacts the way we organize our estimates, and thus it must be established in exact arithmetic. On the other hand, the occurring expressions for each number occupy half a page, and working with them is too tedious (even to MATHEMATICA). Thus we turned to numerical comparison each time we need to see non-coincidence. We must admit that we did not carry any error analysis. Still the values we had to compare were visibly different, in a range above calculation precision error, as we observed for several $A$. Moreover, it is still possible, the way we will explain, to make each check exact.

Consider the Temperley-Lieb category $T L$. Its objects are $T L_{n}=\{1, \ldots, n\}$. Its morphisms are tangles factored by the Kauffman bracket relations (3). Our convention is that composition of morphisms is from bottom to top, and points on $T L_{n}$ are numbered left to right.

These morphisms can be decomposed as a $\mathbb{Z}\left[A^{ \pm 1}\right]$-linear combination of crossingfree arcs. The multiplication of such elements is accomplished by joining top of the first one with bottom of the second, deleting each of the resulting $n$ closed loops, and multiplying by $\left(-A^{2}-A^{-2}\right)^{n}$.

Let us here already fix that we normalize all morphisms by $\left(-A^{3}\right)^{- \text {writhe }}$, to account to the writhe normalization of the bracket, occurring in (1). Note, however, that the writhe of a morphism depends on more the morphism itself, for strand orientation is determined only by the knot diagram as a whole. This writhe normalization will thus be carried out for individual morphisms only because we know in advance the knot diagram made up of all such morphisms taken together.

The normalized closure bracket $c l(E)$ of such an endomorphism $E$ is defined on a crossing-free diagram $D$ as $c l(D)=\left(-A^{2}-A^{-2}\right)^{n-1}$, where $n$ is the number of loops of the braid-style closure of $D$. This is then expanded $\mathbb{Z}\left[A^{ \pm 1}\right]$-linearly.

By (4) the normalized bracket of the tangle closure is then equal to $V\left(A^{-4}\right)$. Let us stipulate that when we write $V$ in the following, we always regard it in $\mathbb{Z}\left[A^{ \pm 1}\right]$ under this substitution $t=A^{-4}$.

We will need a few special morphisms:

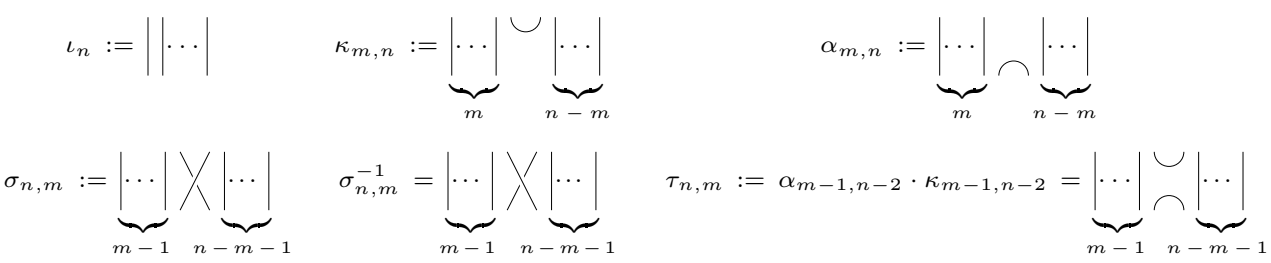

We will in particular deal with $\operatorname{End}\left(T L_{3}\right)$, which is a $\mathbb{Z}\left[A^{ \pm 1}\right]$ module freely gen- 
erated by the 5 basis elements

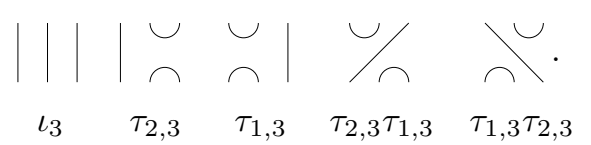

We need to determine $E(T), E\left(T^{\prime}\right)$ coming from $T$ and $T^{\prime}$. Let us fix the following presentation of these tangles, which also gives a numbering of the crossings.

$$
\begin{aligned}
T & :=\kappa_{0,3} \cdot\{-3,2,-3,4,-3,4,2,-3,4,-3,2,-3,4\} \cdot \alpha_{0,3}, \\
T^{\prime}: & =\kappa_{2,3} \cdot\{-1,2,4,-1,-3\} \cdot \alpha_{1,3},
\end{aligned}
$$

where $\left\{i_{1}, i_{2}, \ldots\right\}$ stands for the 5 -braid $\prod_{k} \sigma_{5,\left|i_{k}\right|}^{\operatorname{sgn}\left(i_{k}\right)}$. (Note that the strands in $D_{n}$ are not oriented to point in the same direction within the braids.)

Let $T_{j}$ be $T$ with crossing $j$ switched, and $T_{j}^{\prime}$ be $T^{\prime}$ with crossing $j$ switched.

Since in $D_{n, i, j}$ we have iteration of $T^{\prime}$, we will need to look in particular at $E\left(T^{\prime}\right) \in \operatorname{End}\left(T L_{3}\right)$, the endomorphism of $T L_{3}$ induced by $T^{\prime}$.

It is important to determine the eigenvalues of $E\left(T^{\prime}\right)$.

We found by calculation that (for the values of $A$ we considered) $E\left(T^{\prime}\right)$ has 3 different eigenvalues of different norm. We name them $\lambda=\lambda_{3}, \lambda^{\prime}=\lambda_{2}$ and $\lambda^{\prime \prime}=\lambda_{1}$. So

$$
\left|\lambda^{\prime \prime}\right|<\left|\lambda^{\prime}\right|<|\lambda|
$$

Still $E\left(T^{\prime}\right)$ diagonalizes. This has to be checked in exact arithmetic, since it is a non-generic condition (at the presence of repeated eigenvalues).

By looking at the endomorphisms in the diagonalizing basis of $E\left(T^{\prime}\right)$, we see then that

$$
\begin{gathered}
V\left(D_{n+1,0, c}\right)=\sum_{k=1}^{3} d_{k}^{[c]} \lambda_{k}^{n}, \\
V\left(D_{n_{1}+n_{2}+1, n_{1}+1, c}\right)=\sum_{k=1}^{3} \sum_{l=1}^{3} d_{k, l}^{[c]} \lambda_{k}^{n_{1}} \lambda_{l}^{n_{2}} .
\end{gathered}
$$

The numbers $d_{k}^{[c]}$ and $d_{k, l}^{[c]}$ can be determined in two ways.

The first one is to evaluate the l.h.s. for 3 values of $k$ and $l$. We have

$$
\begin{aligned}
V\left(D_{n+1,0, j}\right) & =\operatorname{cl}\left(\iota_{3} \cdot E\left(T_{j}\right) \cdot E\left(T^{\prime}\right)^{n+1}\right), \\
V\left(D_{n_{1}+n_{2}+1, n_{1}+1, j}\right) & =\operatorname{cl}\left(\iota_{3} \cdot E(T) \cdot E\left(T^{\prime}\right)^{n_{1}} \cdot E\left(T_{j}^{\prime}\right) \cdot E\left(T^{\prime}\right)^{n_{2}}\right) .
\end{aligned}
$$

We also checked that the Jones polynomials are correct by comparing them with a skein calculation.

Then we have to solve a $3 \times 3$ resp. $9 \times 9$ linear system. We employed this method to obtain numerical values for $d_{k}^{[c]}$ and $d_{k, l}^{[c]}$. We used $k, l=0,1,2$, but checked that for some other choices we obtain the same solutions (numerically).

However, it became necessary and useful to determine some $d_{k, l}^{[c]}$ exactly, while handling a $9 \times 9$ system with page-long entries was too daunting to MATHEMATICA. Then we used a different method. 
Let $Q$ be a diagonalizing basis of $E\left(T^{\prime}\right)$, so that

$$
E\left(T^{\prime}\right)=Q \operatorname{diag}\left(\widehat{\lambda}_{i}\right) Q^{-1},
$$

with $\widehat{\lambda}_{i}=\lambda_{j}$ for some $j$. The matrix $Q$ is determined by MATHEMATICA.

Define the 'fake' matrices

$$
F_{j}=Q \operatorname{diag}\left(\delta_{\lambda_{j}, \widehat{\lambda}_{i}}\right) Q^{-1}
$$

where $\delta$ is Kronecker's delta. Then we obtain $d_{k, l}^{[c]}$ by replacing $E\left(T^{\prime}\right)$ in (20) by a fake matrix (normalized as if it were of writhe 0):

$$
d_{k, l}^{[c]}=\operatorname{cl}\left(\iota_{3} \cdot E(T) \cdot F_{k} \cdot E\left(T_{c}^{\prime}\right) \cdot F_{l}\right) .
$$

This yields then (and faster) the exact values for $d_{k, l}^{[c]}$.

This calculation was needed to verify the numerically suggested coincidence

$$
d_{3,3}^{[2]}=d_{3,3}^{[5]} .
$$

Moreover it was very useful to establish exactly that

$$
d_{3,1}^{[c]}=d_{1,3}^{[c]}=0
$$

for all $c=1, \ldots, 5$. (For $A_{0}$ and $d_{1,3}^{[3]}$, MATHEMATICA needed a little manual help along the way.)

The property (21) is the essential reason we chose the values $A_{0}$ and $A_{0}^{\prime}$ for $A$. Apparently there is always this vanishing for some branch of eigenvalues (when $A$ varies continuously), but for many $A$ the $\lambda_{i}$ order in norm so that $d_{3,2}^{[c]}$ and $d_{2,3}^{[c]}$ vanish instead.

With this now we can distinguish $D_{n}$ and its crossing-switched versions $D_{n}^{\prime}$ in cases. Our attitude will be to find a contradiction if some among (the specific evaluations of $) V\left(D_{n}\right)$ and $V\left(D_{n}^{\prime}\right)$ match for arbitrarily large $n$.

Case 1. $D_{n, 0, c}$ among each other for $0 \leq c \leq 13$. (This includes $D_{n}=D_{n, 0,0}$.) By normalizing (17), we obtain

$$
\frac{1}{\lambda^{n}} V\left(D_{n+1,0, c}\right)=d_{3}^{[c]}+d_{2}^{[c]} \cdot\left(\frac{\lambda^{\prime}}{\lambda}\right)^{n}+d_{1}^{[c]} \cdot\left(\frac{\lambda^{\prime \prime}}{\lambda}\right)^{n} .
$$

Since the bases are in norm $<1$, for large $n$ only the comparison of $d_{3}^{[c]}$ matters. We determined that for both $A_{0}$ and $A_{0}^{\prime}$ all $d_{3}^{[c]}$ are different. Here is the list of increasingly ordered norms $\left|d_{3}^{[c]}\right|$ for $A=A_{0}$.

$$
\begin{array}{r}
\{1.74146,2.28564,2.51662,3.60422,4.28911,5.20145,5.32003 \\
5.64902,6.12361,6.6246,7.61719,10.1355,12.0127,13.9591\}
\end{array}
$$

In the following when we say " $n$ large (enough)", we mean some bound $n \geq n_{0}$. The "almost all $n$ " in the theorem will be obtained by combining all these bounds. (They can be made explicit, at the cost of further technicalities.)

Case 2. $D_{n, 0, c^{\prime}}$ from $D_{n, l, c}$ (with $0 \leq c^{\prime} \leq 13$ and $1 \leq c \leq 5$ ). We have, using $O$-notation,

$$
\frac{1}{\lambda^{n}} V\left(D_{n+1, l+1, c}\right)=\widehat{\lambda}(n, l, c)+O\left[\left(\frac{\lambda^{\prime}}{\lambda}\right)^{n}\right]
$$


with

$$
\widehat{\lambda}(n, l, c)=d_{33}^{[c]}+d_{23}^{[c]}\left(\frac{\lambda^{\prime}}{\lambda}\right)^{l}+d_{13}^{[c]}\left(\frac{\lambda^{\prime \prime}}{\lambda}\right)^{l}+d_{32}^{[c]}\left(\frac{\lambda^{\prime}}{\lambda}\right)^{n-l}+d_{31}^{[c]}\left(\frac{\lambda^{\prime \prime}}{\lambda}\right)^{n-l} .
$$

Now fix $c$ and $c^{\prime}$. We determine $l_{0}$ by being the smallest nonnegative integer with

$$
\left|d_{33}^{[c]}-d_{3}^{\left[c^{\prime}\right]}\right|>\left(\left|d_{23}^{[c]}\right|+\left|d_{32}^{[c]}\right|\right)\left|\frac{\lambda^{\prime}}{\lambda}\right|^{l_{0}}+\left(\left|d_{13}^{[c]}\right|+\left|d_{31}^{[c]}\right|\right)\left|\frac{\lambda^{\prime \prime}}{\lambda}\right|^{l_{0}}
$$

(we tested this numerically with an extra tolerance of $10^{-5}$ ).

It is clear that when $l \geq l_{0}$ and $n-l \geq l_{0}$, we have $\widehat{\lambda}(n, l, c) \neq d_{3}^{\left[c^{\prime}\right]}$. This leads then to the distinctness of $V\left(D_{n, l, c}\right)$ and $V\left(D_{n, 0, c^{\prime}}\right)$ for large $n$ in the following way.

When $n \rightarrow \infty$ and we have a sequence $l=l(n)$ with $l \geq l_{0}$ and $n-l \geq l_{0}$, then $\frac{1}{\lambda^{n}} V\left(D_{n, l, c}\right)$ is distinct from $\frac{1}{\lambda^{n}} V\left(D_{n, 0, c^{\prime}}\right)$ for $n \geq n_{0}$. Here $n_{0}$ depends on the difference between the hand-sides of (22) but not on the concretely chosen $l(n)$.

It remains now to test the cases $l<l_{0}$ and $n-l<l_{0}$. We can then w.l.o.g. assume that $l$ or $n-l$ is fixed. Let us define

$$
\lambda(l, c):=d_{33}^{[c]}+d_{23}^{[c]}\left(\frac{\lambda^{\prime}}{\lambda}\right)^{l}+d_{13}^{[c]}\left(\frac{\lambda^{\prime \prime}}{\lambda}\right)^{l}, \quad \tilde{\lambda}(l, c):=d_{33}^{[c]}+d_{32}^{[c]}\left(\frac{\lambda^{\prime}}{\lambda}\right)^{l}+d_{31}^{[c]}\left(\frac{\lambda^{\prime \prime}}{\lambda}\right)^{l} .
$$

These are the limits of $\frac{1}{\lambda^{n}} V\left(D_{n, l, c}\right)$ when either $l$ or $n-l$ is fixed, and $n \rightarrow \infty$.

It is enough to test now that

$$
\lambda(l, c) \neq d_{3}^{\left[c^{\prime}\right]} \text { and } \widetilde{\lambda}(l, c) \neq d_{3}^{\left[c^{\prime}\right]} \text { for } 0 \leq l<l_{0} .
$$

Each of these finitely many non-coincidences gives then some $n_{0}$ such that $V\left(D_{n, l, c}\right) \neq$ $V\left(D_{n, 0, c^{\prime}}\right)$ for $n \geq n_{0}$.

For $A_{0}$ the smallest norm of difference of the hand-sides of (24), in the instances necessary to test, is $>1$. For $A_{0}^{\prime}$ it is $\approx 0.162$.

Case 3. The distinction of $D_{n, l, c}$ and $D_{n, l^{\prime}, c^{\prime}}$ is more complicated, and requires several subcases.

Case 3.1. $d_{33}^{[c]} \neq d_{33}^{\left[c^{\prime}\right]}$. Fix such a $c, c^{\prime}$.

We determine $l_{0}$ by being the smallest nonnegative integer with

$$
\left|d_{33}^{[c]}-d_{33}^{\left[c^{\prime}\right]}\right|>\left(\left|d_{23}^{[c]}\right|+\left|d_{32}^{[c]}\right|\right)\left|\frac{\lambda^{\prime}}{\lambda}\right|^{l_{0}}+\left(\left|d_{13}^{[c]}\right|+\left|d_{31}^{[c]}\right|\right)\left|\frac{\lambda^{\prime \prime}}{\lambda}\right|^{l_{0}}
$$

(we tested this numerically with an extra tolerance of $10^{-5}$ ).

It is then enough to prove that with (23),

$$
\{\lambda(l, c), \widetilde{\lambda}(l, c)\} \cap\left\{\lambda\left(l^{\prime}, c^{\prime}\right), \widetilde{\lambda}\left(l^{\prime}, c^{\prime}\right)\right\}=\varnothing
$$

for $0 \leq l<l_{0}$ and $l^{\prime} \geq 0$ arbitrary.

For such $l$ let $l_{1}=l_{1}(l)$ be the smallest nonnegative integer with

$$
\left|d_{33}^{\left[c^{\prime}\right]}-\lambda(l, c)\right|>\left(\left|d_{23}^{\left[c^{\prime}\right]}\right|+\left|d_{32}^{\left[c^{\prime}\right]}\right|\right)\left|\frac{\lambda^{\prime}}{\lambda}\right|^{l_{1}}+\left(\left|d_{13}^{\left[c^{\prime}\right]}\right|+\left|d_{31}^{\left[c^{\prime}\right]}\right|\right)\left|\frac{\lambda^{\prime \prime}}{\lambda}\right|^{l_{1}}
$$


resp. with

$$
\left|d_{33}^{\left[c^{\prime}\right]}-\tilde{\lambda}(l, c)\right|>\left(\left|d_{23}^{\left[c^{\prime}\right]}\right|+\left|d_{32}^{\left[c^{\prime}\right]}\right|\right)\left|\frac{\lambda^{\prime}}{\lambda}\right|^{l_{1}}+\left(\left|d_{13}^{\left[c^{\prime}\right]}\right|+\left|d_{31}^{\left[c^{c^{\prime}}\right]}\right|\right)\left|\frac{\lambda^{\prime \prime}}{\lambda}\right|^{l_{1}}
$$

(we tested this numerically with an extra tolerance of $10^{-5}$ ).

Then (26) holds for $l^{\prime} \geq l_{1}$ for almost all $n$ (where 'almost' is uniform in $l^{\prime}$ ). We finish the check by testing (26) for $0 \leq l<l_{0}$ and $0 \leq l^{\prime}<l_{1}(l)$.

The smallest norm of difference in non-coincidence tests is about 0.034 for $A_{0}$ and about 0.0479 for $A_{0}^{\prime}$.

Case 3.2. $c=c^{\prime}$. This case is now more complicated, because the leading terms of $\frac{1}{\lambda^{n}} V\left(D_{n, l, c}\right)$ and $\frac{1}{\lambda^{n}} V\left(D_{n, l^{\prime}, c}\right)$ on the right of (18) are equal.

We will assume w.l.o.g. $l^{\prime}>l \geq 0$ and examine the difference, which we now organize thus:

$$
\text { (29) } \frac{1}{\lambda^{n}}\left(V\left(D_{n+1, l+1, c}\right)-V\left(D_{n+1, l^{\prime}+1, c}\right)\right)=d_{2,3}^{[c]} \cdot(A) \cdot\left(\frac{\lambda^{\prime}}{\lambda}\right)^{l}-d_{3,2}^{[c]} \cdot(B) \cdot\left(\frac{\lambda^{\prime}}{\lambda}\right)^{n-l^{\prime}} \text {, }
$$

where

$$
\begin{aligned}
(A)=1-\left(\frac{\lambda^{\prime}}{\lambda}\right)^{l^{\prime}-l}+\frac{d_{13}^{[c]}}{d_{2,3}^{[c]}}\left[\left(\frac{\lambda^{\prime \prime}}{\lambda^{\prime}}\right)^{l}-\left(\frac{\lambda^{\prime \prime}}{\lambda^{\prime}}\right)^{l^{\prime}} \cdot\left(\frac{\lambda^{\prime}}{\lambda}\right)^{l^{\prime}-l}\right]+ \\
\frac{d_{12}^{[c]}}{d_{2,3}^{[c]}}\left[\left(\frac{\lambda^{\prime \prime}}{\lambda^{\prime}}\right)^{l} \cdot\left(\frac{\lambda^{\prime}}{\lambda}\right)^{n-l}-\left(\frac{\lambda^{\prime \prime}}{\lambda^{\prime}}\right)^{l^{\prime}} \cdot\left(\frac{\lambda^{\prime}}{\lambda}\right)^{n-l^{\prime}} \cdot\left(\frac{\lambda^{\prime}}{\lambda}\right)^{l^{\prime}-l}\right]+ \\
\frac{d_{11}^{[c]}}{d_{2,3}^{[c]}}\left[\left(\frac{\lambda^{\prime \prime}}{\lambda^{\prime}}\right)^{l} \cdot\left(\frac{\lambda^{\prime \prime}}{\lambda}\right)^{n-l}-\left(\frac{\lambda^{\prime \prime}}{\lambda^{\prime}}\right)^{l^{\prime}} \cdot\left(\frac{\lambda^{\prime \prime}}{\lambda}\right)^{n-l^{\prime}} \cdot\left(\frac{\lambda^{\prime}}{\lambda}\right)^{l^{\prime}-l}\right]
\end{aligned}
$$

and

$$
\begin{aligned}
& (B)=1-\left(\frac{\lambda^{\prime}}{\lambda}\right)^{l^{\prime}-l}+\frac{d_{31}^{[c]}}{d_{3,2}^{[c]}}\left[\left(\frac{\lambda^{\prime \prime}}{\lambda^{\prime}}\right)^{n-l^{\prime}}-\left(\frac{\lambda^{\prime \prime}}{\lambda^{\prime}}\right)^{n-l} \cdot\left(\frac{\lambda^{\prime}}{\lambda}\right)^{l^{\prime}-l}\right]+ \\
& \frac{d_{22}^{[c]}}{d_{3,2}^{[c]}}\left[\left(\frac{\lambda^{\prime}}{\lambda}\right)^{l^{\prime}}-\left(\frac{\lambda^{\prime}}{\lambda}\right)^{l} \cdot\left(\frac{\lambda^{\prime}}{\lambda}\right)^{l^{\prime}-l}\right]+ \\
& \frac{d_{21}^{[c]}}{d_{3,2}^{[c]}}\left[\left(\frac{\lambda^{\prime}}{\lambda}\right)^{l^{\prime}} \cdot\left(\frac{\lambda^{\prime \prime}}{\lambda^{\prime}}\right)^{n-l^{\prime}}-\left(\frac{\lambda^{\prime}}{\lambda}\right)^{l} \cdot\left(\frac{\lambda^{\prime \prime}}{\lambda^{\prime}}\right)^{n-l} \cdot\left(\frac{\lambda^{\prime}}{\lambda}\right)^{l^{\prime}-l}\right] .
\end{aligned}
$$

Note that when applying (18) in (29), the terms with $d_{11}$ and $d_{22}$ depend only on $n$ but not on $l$, and thus cancel out in the difference. We did not ignore them, since we will adapt this case to case 3.3 below, and there the terms will receive a non-zero factor.

Now the first written term ' 1 ' in (A) and (B) is the most important. Let us consider the case that it is dominant.

Let $l_{0}$ be the minimal nonnegative integer with

$$
\begin{aligned}
& 1-\left|\frac{\lambda^{\prime}}{\lambda}\right|-2 \frac{\left|d_{13}^{[c]}\right|+\left|d_{12}^{[c]}\right|}{\left|d_{23}^{[c]}\right|}\left|\frac{\lambda^{\prime \prime}}{\lambda^{\prime}}\right|^{l_{0}}>0 \quad \text { and } \\
& 1-\left|\frac{\lambda^{\prime}}{\lambda}\right|-2 \frac{\left|d_{31}^{[c]}\right|+\left|d_{21}^{[c]}\right|}{\left|d_{32}^{[c]}\right|}\left|\frac{\lambda^{\prime \prime}}{\lambda^{\prime}}\right|^{l_{0}}>0 .
\end{aligned}
$$


Case 3.2.1. This means then that for $l, n-l^{\prime} \geq l_{0}$ we have $|(A)|>\varepsilon$ and $|(B)|>\varepsilon$, where $\varepsilon$ is independent of $n, l, l^{\prime}$. On the other hand, it is straightforward that there are constants $a, b$ independent of $n, l$ with

$$
|(A)|<a
$$

and

$$
|(B)|<b .
$$

Then with (34) and (35) if

$$
d_{2,3}^{[c]} \cdot(A) \cdot\left(\frac{\lambda^{\prime}}{\lambda}\right)^{l}=d_{3,2}^{[c]} \cdot(B) \cdot\left(\frac{\lambda^{\prime}}{\lambda}\right)^{n-l^{\prime}},
$$

then we have for $l, n-l^{\prime} \geq l_{0}$

$$
\frac{1}{\varepsilon^{\prime}}>\frac{\left|d_{2,3}^{[c]}\left(\frac{\lambda^{\prime}}{\lambda}\right)^{l}\right|}{\left|d_{3,2}^{[c]}\left(\frac{\lambda^{\prime}}{\lambda}\right)^{n-l^{\prime}}\right|}>\varepsilon^{\prime} .
$$

Thus

$$
\left|\left(n-l^{\prime}\right)-l\right| \leq C
$$

Here $C$ involves the constants $a, b$ and $\varepsilon$, and a logarithm of $\left|\frac{d_{2,3}^{[c]}}{d_{3,2}^{[c]}}\right|$, and is independent of $n, l, l^{\prime}$.

This then implies that $l$ and $n-l^{\prime}$ are both bounded or both unbounded as $n \rightarrow \infty$.

Case 3.2.1.1. If $l$ and $n-l^{\prime}$ are bounded, then we can assume them fixed. Then taking the limit of the l.h.s. of (29) for $n \rightarrow \infty$ and using (18), we get to exclude an equality of the type

$$
d_{2,3}^{[c]}\left(\frac{\lambda^{\prime}}{\lambda}\right)^{l}+d_{1,3}^{[c]}\left(\frac{\lambda^{\prime \prime}}{\lambda}\right)^{l}=d_{3,2}^{[c]}\left(\frac{\lambda^{\prime}}{\lambda}\right)^{n-l^{\prime}}+d_{3,1}^{[c]}\left(\frac{\lambda^{\prime \prime}}{\lambda}\right)^{n-l^{\prime}} .
$$

The examination of this is somewhat tedious, which is why we have prepared $(21)$. Then excluding (37) blows down to saying that $\frac{d_{2,3}^{[c]}}{d_{3,2}^{[c]}}$ is not an integral power of $\frac{\lambda^{\prime}}{\lambda}$, which is directly checked (already by using norms).

Note that still (21), which we do not know to hold for all tangles, is not indispensable (and this is the reason we have not simplified the above formulas using it). For larger $l, n-l^{\prime}$ we can estimate the second terms on either hand sides of (37) against the first, and approximate the contradiction for $d_{13}=d_{31}=0$. For a few smaller $l$ or $n-l^{\prime}$ that may remain, we can examine (37) ad hoc. 
Case 3.2.1.2. Now let $l$ and $n-l^{\prime}$ be unbounded. Then for $n \rightarrow \infty$ the $d_{22}^{[c]}$-term in (31) becomes negligible along with the $d_{11}, d_{12}$ and $d_{21}$ terms, and

$$
\lim _{n \rightarrow \infty} \frac{(B)-(A)}{(A)}=\lim _{n \rightarrow \infty} \frac{\frac{d_{22}^{[c]}}{d_{32}^{[c]}}\left[\left(\frac{\lambda^{\prime}}{\lambda}\right)^{l^{\prime}}-\left(\frac{\lambda^{\prime}}{\lambda}\right)^{l}\right]}{1-\left(\frac{\lambda^{\prime}}{\lambda}\right)^{l-l^{\prime}}}=0 .
$$

Now from (36) we have

$$
d_{23}^{[c]}=d_{32}^{[c]} \cdot\left(\frac{\lambda^{\prime}}{\lambda}\right)^{n-l^{\prime}-l} \cdot \frac{(B)}{(A)} .
$$

When we let $n \rightarrow \infty$, we have that the second factor on the right takes only finitely many (non-zero) values, while the third converges to 1 .

We obtain then again that $\frac{d_{23}^{[c]}}{d_{32}^{[c]}}$ must be some integral power of $\frac{\lambda^{\prime}}{\lambda}$, which we already saw is not the case.

Case 3.2.2. After this we still have to deal with $l<l_{0}$ or $0 \leq n-l^{\prime}<l_{0}$. We can then assume $l$ resp. $n-l^{\prime}$ is fixed, and work as in case 3.1 with (27) and (28).

Case 3.3. $c \neq c^{\prime}$ but $d_{33}^{[c]}=d_{33}^{\left[c^{\prime}\right]}$. This final case appears non-generic, and is an unpleasant feature of the tangle $T^{\prime}$ we work with. It occurs for $c, c^{\prime}=2,5$.

The case is treated parallelly to case 3.2. We assume again $l \leq l^{\prime}$ and must take both orderings of $c, c^{\prime}$. Then (32) and (33) become, with $\tilde{l}=l^{\prime}-l$,

$$
\left(\left.\min _{\tilde{l} \geq 0}|1-| \frac{d_{23}^{\left[c^{\prime}\right]}}{d_{23}^{[c]}}|| \frac{\lambda^{\prime}}{\lambda}\right|^{\tilde{l}} \mid\right)-\frac{\left|d_{13}^{[c]}\right|+\left|d_{12}^{[c]}\right|+\left|d_{13}^{\left[c^{\prime}\right]}\right|+\left|d_{12}^{\left[c^{\prime}\right]}\right|+\left|d_{11}^{[c]}-d_{11}^{\left[c^{\prime}\right]}\right|}{\left|d_{23}^{[c]}\right|}\left|\frac{\lambda^{\prime \prime}}{\lambda^{\prime}}\right|^{l_{0}}>0
$$

and

$$
\left(\left.\min _{\tilde{l} \geq 0}|1-| \frac{d_{32}^{\left[c^{\prime}\right]}}{d_{32}^{[c]}}|| \frac{\lambda^{\prime}}{\lambda}\right|^{\tilde{i}} \mid\right)-\frac{\left|d_{31}^{[c]}\right|+\left|d_{21}^{[c]}\right|+\left|d_{31}^{\left[c^{\prime}\right]}\right|+\left|d_{21}^{\left[c^{\prime}\right]}\right|}{\left|d_{32}^{[c]}\right|}\left|\frac{\lambda^{\prime \prime}}{\lambda^{\prime}}\right|^{l_{0}}-\frac{\left|d_{22}^{[c]}-d_{22}^{\left[c^{\prime}\right]}\right|}{\left|d_{32}^{[c]}\right|}\left|\frac{\lambda^{\prime}}{\lambda}\right|^{l_{0}}>0
$$

(plus the two inequalities obtained by interchanging $c$ and $c^{\prime}$ ). And then we must ascertain that

$$
g_{1}=\frac{d_{32}^{[c]}}{d_{23}^{\left[c^{\prime}\right]}}, \quad g_{2}=\frac{d_{32}^{\left[c^{\prime}\right]}}{d_{23}^{[c]}}
$$

are not integral powers of $\lambda^{\prime} / \lambda$.

We have for $A=A_{0}^{\prime}$ that $\left|g_{i}\right| \approx 2.636$ and $\left|\lambda / \lambda^{\prime}\right| \approx 2.203$, and for $A=A_{0}$ that $\left|g_{i}\right| \approx 1.151$ and $\left|\lambda / \lambda^{\prime}\right| \approx 2.632$.

Again for $\min \left(l, n-l^{\prime}\right)<l_{0}$ we would need to test some finite number of noncoincidences of the sort (26). However, this case in fact does not occur, since $l_{0}=0$ (for both $A_{0}$ and $A_{0}^{\prime}$ ).

3.3. A non-alternating series of examples. Shinjo and Taniyama also proposed an infinite sequence of examples of non-alternating diagrams.

In this case

$$
T^{\prime}=\kappa_{0,3} \cdot\{3,2,-3,-4,-3\} \cdot \alpha_{1,3} \quad \text { and } \quad T=T^{\prime} \cdot \kappa_{0,3} \cdot\{3,4,-3,4,-3,2,-3,4,-3\} \cdot \alpha_{1,3} \text {. }
$$


Note that the extra copy of $T^{\prime}$ inside $T$ needs a little attention, since the orientation of strands is different from the others.

Again let $D_{n}$ be the closure of $T \cdot\left(T^{\prime}\right)^{n}$.

THEOREM 4. For almost all $n$, the diagram $D_{n}$ is everywhere different.

Proof. We repeat the proof of theorem 3 with a few modifications.

Everything until and including case 3.1 goes through for a proper value of $A$ (we specify below).

Case 3.2 works as before for $c=1,2,4$. However, for $c=3,5$ we have

$$
d_{32}^{[3]}=d_{23}^{[5]}=0
$$

(which must be checked in exact arithmetic). The properties (21) hold again for all $c=1, \ldots, 5$.

Let us assume $l^{\prime}>l$. Now with (21) and (39) we have for $c=3$

$$
\begin{aligned}
& \frac{1}{\lambda^{n}}\left(V\left(D_{n+1, l+1, c}\right)-V\left(D_{n+1, l^{\prime}+1, c}\right)\right) \\
= & \left(\frac{\lambda^{\prime}}{\lambda}\right)^{l} \cdot\left\{d_{23}^{[c]}\left[1-\left(\frac{\lambda^{\prime}}{\lambda}\right)^{l^{\prime}-l}\right]+d_{21}^{[c]}\left[\left(\frac{\lambda^{\prime \prime}}{\lambda}\right)^{n-l}-\left(\frac{\lambda^{\prime}}{\lambda}\right)^{l^{\prime}-l}\left(\frac{\lambda^{\prime \prime}}{\lambda}\right)^{n-l^{\prime}}\right]\right. \\
& \left.+d_{12}^{[c]}\left[\left(\frac{\lambda^{\prime \prime}}{\lambda^{\prime}}\right)^{l}\left(\frac{\lambda^{\prime}}{\lambda}\right)^{n-l}-\left(\frac{\lambda^{\prime}}{\lambda}\right)^{l^{\prime}-l}\left(\frac{\lambda^{\prime \prime}}{\lambda^{\prime}}\right)^{l^{\prime}}\left(\frac{\lambda^{\prime}}{\lambda}\right)^{n-l^{\prime}}\right]\right\} .
\end{aligned}
$$

The $d_{12}^{[c]}$-term becomes negligible. Thus, in case of vanishing of (40), when we divide by $\left(\frac{\lambda^{\prime}}{\lambda}\right)^{l}$ and take the limit for $n \rightarrow \infty$, we have

$$
d_{23}^{[c]}\left[1-\left(\frac{\lambda^{\prime}}{\lambda}\right)^{l^{\prime}-l}\right]=-d_{21}^{[c]}\left[\left(\frac{\lambda^{\prime \prime}}{\lambda}\right)^{n-l}-\left(\frac{\lambda^{\prime}}{\lambda}\right)^{l^{\prime}-l}\left(\frac{\lambda^{\prime \prime}}{\lambda}\right)^{n-l^{\prime}}\right] .
$$

Now $l^{\prime}-l>0$ and $n-l^{\prime} \geq 0$. If some of these two quantities becomes unbounded for $n \rightarrow \infty$, the r.h.s. goes to 0 , while the l.h.s. cannot. Thus we can w.l.o.g. assume that $l^{\prime}-l$ and $n-l^{\prime}$ are fixed.

We determine $l_{0}$ by being the smallest nonnegative integer with

$$
\left|d_{23}^{[c]}\right|\left(1-\left|\frac{\lambda^{\prime}}{\lambda}\right|^{l_{0}}\right)>2\left|d_{21}^{[c]}\right|\left|\frac{\lambda^{\prime}}{\lambda}\right|^{l_{0}}
$$

(we tested this numerically with an extra tolerance of $10^{-5}$ ). Then $\tilde{l}:=l^{\prime}-l<l_{0}$. For each such $\tilde{l}$ consider all $k:=n-l^{\prime} \geq 0$ with

$$
\left|d_{23}^{[c]}\right|\left(1-\left|\frac{\lambda^{\prime}}{\lambda}\right|^{\tilde{l}}\right) \leq 2\left|d_{21}^{[c]}\right|\left|\frac{\lambda^{\prime}}{\lambda}\right|^{\tilde{l}}\left|\frac{\lambda^{\prime \prime}}{\lambda}\right|^{k},
$$

and test the norm of

$$
d_{23}^{[c]}\left[1-\left(\frac{\lambda^{\prime}}{\lambda}\right)^{\tilde{l}}\right]+d_{21}^{[c]}\left[\left(\frac{\lambda^{\prime \prime}}{\lambda}\right)^{\tilde{l}+k}-\left(\frac{\lambda^{\prime}}{\lambda}\right)^{\tilde{l}}\left(\frac{\lambda^{\prime \prime}}{\lambda}\right)^{k}\right]
$$


to be sufficiently away from 0 .

If $d_{23}^{[c]}=0$ (as for $c=5$ here), then in the test we just described replace $d_{23}^{[c]}$ by $d_{32}^{[c]}$ and $d_{21}^{[c]}$ by $d_{12}^{[c]}$.

This worked out for $c=3,5$.

Case 3.3 occurs now for $c, c^{\prime}=3,5$. These are exactly the values involved in (39). Let $c=3$ and $c^{\prime}=5$, and make no restriction on how $l$ and $l^{\prime}$ compare.

The coincidence we have to exclude now can be written thus:

$$
\begin{aligned}
&(42) \quad\left(\frac{\lambda^{\prime}}{\lambda}\right)^{l} d_{23}^{[c]}\{1+\frac{d_{22}^{[c]}}{d_{23}^{[c]}}\left(\frac{\lambda^{\prime}}{\lambda}\right)^{n-l}+\frac{d_{21}^{[c]}}{d_{23}^{[c]}}\left(\frac{\lambda^{\prime \prime}}{\lambda}\right)^{n-l} \\
&\left.+\frac{d_{12}^{[c]}}{d_{23}^{[c]}}\left(\frac{\lambda^{\prime}}{\lambda}\right)^{n-l}\left(\frac{\lambda^{\prime \prime}}{\lambda^{\prime}}\right)^{l}+\frac{d_{11}^{[c]}}{d_{23}^{[c]}}\left(\frac{\lambda^{\prime}}{\lambda}\right)^{n-l}\left(\frac{\lambda^{\prime \prime}}{\lambda^{\prime}}\right)^{n}\right\} \\
&(43)=\left(\frac{\lambda^{\prime}}{\lambda}\right)^{n-l^{\prime}} d_{32}^{\left[c^{\prime}\right]}\left\{1+\frac{d_{22}^{\left[c^{\prime}\right]}}{d_{32}^{\left[c^{\prime}\right]}}\left(\frac{\lambda^{\prime}}{\lambda}\right)^{l^{\prime}}+\frac{d_{12}^{\left[c^{\prime}\right]}}{d_{32}^{\left[c^{\prime}\right]}}\left(\frac{\lambda^{\prime \prime}}{\lambda}\right)^{l^{\prime}}\right. \\
&\left.+\frac{d_{21}^{\left[c^{\prime}\right]}}{d_{32}^{\left[c^{\prime}\right]}}\left(\frac{\lambda^{\prime}}{\lambda}\right)^{l^{\prime}}\left(\frac{\lambda^{\prime \prime}}{\lambda^{\prime}}\right)^{n-l^{\prime}}+\frac{d_{11}^{\left[c^{\prime}\right]}}{d_{32}^{\left[c^{\prime}\right]}}\left(\frac{\lambda^{\prime}}{\lambda}\right)^{l^{\prime}}\left(\frac{\lambda^{\prime \prime}}{\lambda^{\prime}}\right)^{n}\right\} .
\end{aligned}
$$

This is rather daunting to work with. However, it drastically simplifies by checking (exactly, after numerical evidence) that in our case

$$
d_{22}^{[3]}=d_{22}^{[5]} \quad \text { and } \quad d_{21}^{[3]}=d_{12}^{[5]}=0 .
$$

This means that the terms that involve $d_{22}^{[c]}, d_{21}^{[c]}$ in (42) and those with $d_{22}^{\left[c^{\prime}\right]}, d_{12}^{\left[c^{\prime}\right]}$ in (43) drop out.

The other terms in the braces different from 1 become in norm smaller than 1 for $n \geq n_{0}$ independently of $l$ or $l^{\prime}$. Thus for $n$ large enough we see again that $\left|n-l^{\prime}-l\right|$ is bounded (independently of $n, l$ and $l^{\prime}$ ). Then, however, letting $n \rightarrow \infty$, we obtain again that $g_{2}$ in (38) must be an integral power of $\lambda^{\prime} / \lambda$, which is easily excluded.

The values of $A$ we worked with are $\frac{15}{16}-s \cdot \sqrt{-1}$, where $s$ is one of $\frac{2}{13}, \frac{1}{7}, \frac{1}{8}, \frac{1}{10}$ and $\frac{1}{16}$. For every of the above cases the smallest norm of difference that had to be tested was at least 0.01 for some of these five values of $A$.

The delicate choice of values for $A$ was made necessary by the way we designed our estimates. If the estimates are properly reorganized, it appears that many more $A$ (and such with even more convenient numerical behavior) can be used. It is evident that this approach, with a certain amount of flexibility in details, can be applied to a rather general iterated tangle construction.

\section{Everywhere equivalent diagrams.}

4.1. Generalities and outline. In order to express ourselves succinctly, let throughout in the following $D$ be a knot diagram and $D^{\prime}$ denote a diagram obtained from $D$ by (exactly) one crossing change.

Following Taniyama, $D$ is called everywhere trivial (resp. everywhere non-trivial) if all $D^{\prime}$ represent (resp. do not represent) the unknot. Everywhere trivial diagrams are a special case of Taniyama's everywhere equivalent (EE) diagrams D, which are 
characterized by demanding that all $D^{\prime}$ should represent the same (though not necessarily trivial) knot. Let us fix here that equivalence means taking mirroring into account, but ignoring orientation. This notion is opposite to that of everywhere different diagrams, except that we did not specify whether $D$ and $D^{\prime}$ should have the same knot type. If they do, let us call $D$ strongly everywhere equivalent (SEE), otherwise weakly everywhere equivalent (WEE).

We will generally assume that prime diagrams are considered up to taking the mirror image. This is done when examining some sort of EE property, which is invariant under taking the mirror image. Thus, for example, a positive diagram below should mean a positive or negative one. We should still emphasize again that links depicted by diagrams $D^{\prime}$ are not considered equivalent in the treatment of EE if they are mirror images. For example, the 8 crossing diagrams of the knots $8_{3}$ and $8_{18}$ in [Ro, appendix] are not EE in our sense.

In the following, we will examine SEE and WEE diagrams, first starting with everywhere trivial diagrams, continuing our preceding work [SA] (proposition 2). We then go over to treat the general case and present several constructions of everywhere equivalent knot diagrams. We speculate (likely too optimistically in question 1) that these constructions give in fact all EE diagrams, and prove (theorem 5) that this is so among diagrams of low genus.

4.2. A sequence of everywhere trivial diagrams. It is not known what are the everywhere trivial diagrams of a non-trivial knot. The following six examples, consisting of two trefoil and four figure-8-knot diagrams, were given in (11) and (12) of [SA] (where "trivial" was called "1-trivial"):

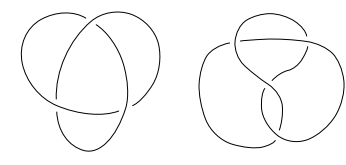

trefoil
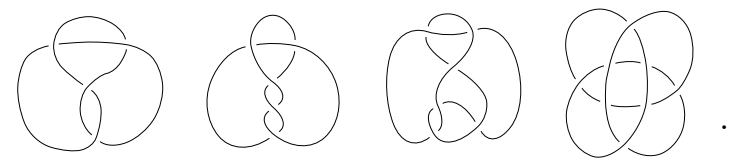

figure-8-knot

It remains an open question whether these are all. (A verification in [SA] up to 14 crossings found no further diagrams.) Theorem 5 below will answer affirmatively the question for diagrams up to genus 3 .

For everywhere trivial diagrams of the unknot, many more examples were found; see $\S 5.2 .2$ of $[\mathrm{SA}]$. There are trivial ways of building more complicated ones by connected sum. (This includes adding kinks, i.e. crossings created or reduced by a Reidemeister I move.)

For prime diagrams (such which are no connected sum) it was observed in [SA] that many of the examples can be extended to infinite families by adding trivial (resolved) clasps. It was then asked (Question 5.6 ibid.) about prime everywhere trivial diagrams of the unknot without trivial clasps.

Here first we observe an answer to that question in analogy to a construction of everywhere non-trivial unknot diagrams shown to me by Shinjo and Taniyama.

Proposition 2. For every crossing number $\geq 11$ there are prime everywhere trivial unknot diagrams without a trivial clasp.

Proof. Look at the (3-string) tangle $T$ on the left of figure 6 . For a knot diagram $D$ containing this tangle, let $D_{k}$ be a diagram obtained by replacing $T$ in $D$ by $T^{k}$, where $T^{k}$ is obtained by composing $k$ copies of $T$. Hereby strings are connected from left to right, as on the right of the figure (for $k=2$ ). 


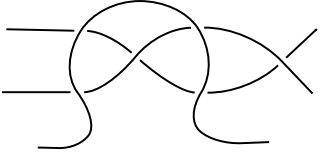

$T$

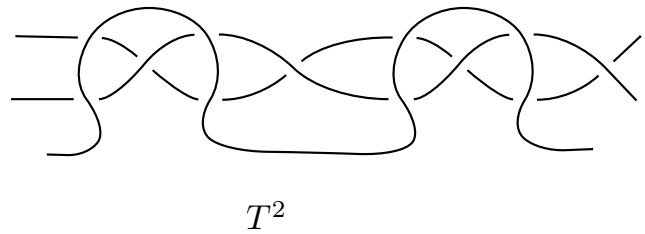

FIG. 6.

Since $T$ simplifies to a trivial tangle diagram (three parallel non-intersecting strings), switching a crossing in one of the $k$ copies of $T$ in $T^{k}$ gives the same tangle as if this crossing is switched in $T$ itself. Thus the set of knot types of diagrams $D_{k}^{\prime}$ is the same as for $D^{\prime}=D_{1}^{\prime}$. In particular, when $D$ is everywhere trivial, so is $D_{k}$.

It turns out that one of the (prime, with no trivial clasps) everywhere trivial diagrams $D$ of the unknot shown in Example 5.4 of [SA], the one with 12 crossings, contains this tangle. By applying the aforementioned argument on this 12 crossing unknot diagram $D$, we obtain a family of everywhere trivial unknot diagrams $D_{k}$ of $6+6 k$ crossings $(k \geq 1)$ with no trivial clasps.

With a minor modification, this idea can be applied also to any crossing changed version of the tangle diagram $T$ in figure 6 which simplifies to a trivial tangle diagram. In particular, the strand going on top in $T$ may pass below the other two strands, and/or these two strands' crossings may be (simultaneously) switched. The 11 and 13 crossing diagrams of Example 5.4 of [SA] contain such tangles.

It remains to find similar diagrams of 14 to 16 crossings. Three suitable diagrams are shown in figure 7. (They still required some computation; in particular the 15 crossing diagram shown is the only one we found.)
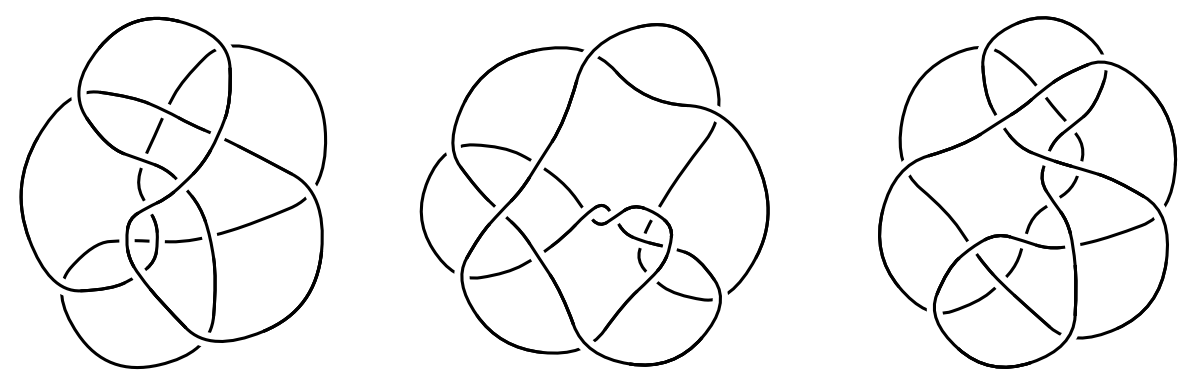

FIG. 7.

4.3. Some families of everywhere equivalent diagrams. We turn now to the more general family of everywhere equivalent (EE) diagrams. The case that $D$ and $D^{\prime}$ are both unknotted suggests itself as too abundant to have an interesting (complete) description. We have made some experiment in the case that some of $D$ or $D^{\prime}$ is knotted. It helped us observe the following series of diagrams. The proof that they are everywhere equivalent is rather straightforward by observation. 
Let $P(p, q)$ be the pretzel tangle diagram $P(p, q)=(\underbrace{p, p, \ldots, p}_{q \text { times }})$.

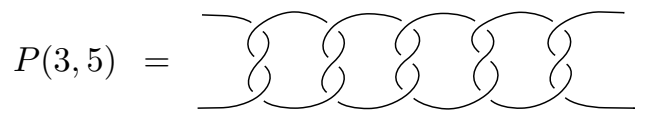

Proposition 3. The diagrams of these types are everywhere equivalent.

1. The pretzel knot diagram $\hat{P}(p, q)$ with $p \geq 1, q \geq 3$ both odd (obtained from $P(p, q)$ as in (45) by closing the two top and two bottom ends).

2. In the following $k \geq 2$.

2.a. The diagram $Q(k, l)$ of the closed 3-braid $\left(\sigma_{1}^{l} \sigma_{2}^{l}\right)^{k}$ for $l$ odd and $3 \nmid k$, and

2.b. the diagram of the closed braid $\left(\sigma_{1} \sigma_{2}\right)^{k}$, in which each crossing is replaced (with orientation possibly different from the braid and adjusted to the resulting diagram) by $l$ positive half-twists in the direction not coinciding with the one of the braid. We assume here $l \geq 1$, and $3 \nmid k$ for $l$ odd. Let this diagram be called $\tilde{Q}(k, l)$.

3. The arborescent diagram

$$
(P(3, p), \cdots, P(3, p))
$$

with $q$ copies of $P(3, p)$ for $p, q \geq 3$ odd.

4. A diagram obtained from those in type 2 by replacing each twist of $l$ crossings by $P(3, l)$ for $l \geq 2$. (This replacement should be done respecting the direction of the twists, as shown below in (47).)

$(47)$
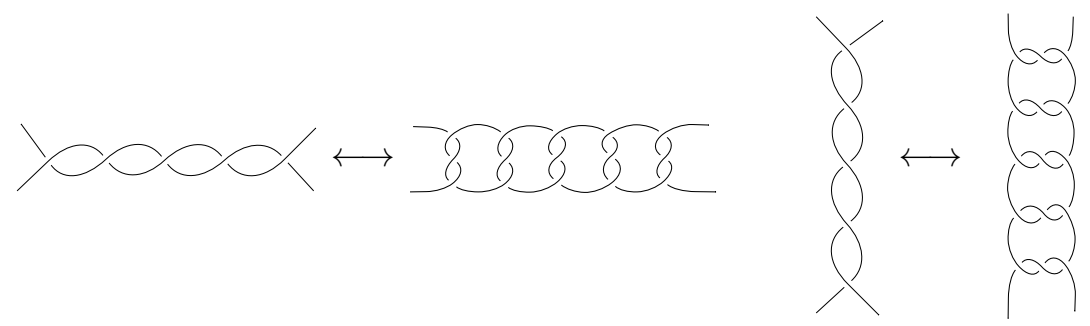

A natural question about such constructions is how exhaustive they are. In the realm of examining this question beyond brute-force verification, we decided below to concentrate on a(n infinite) class of diagrams of low genus studied in [St5, St6, SV]. The work therein allows us to develop here a method for deciding the everywhere equivalence for such diagrams.

We thus recall the preparations collected in $\S 2.9$ regarding generators, as well as the brief explanation about low-degree Vassiliev invariants in $§ 2.7$.

In the following it is legitimate to consider a generator up to flypes. This is because one can easily see that the proofs in $\S 4$ are not affected by mutations. Lowdegree Vassiliev invariants are mutation invariant, and so is the property of a diagram to be unknotted. Also, the diagrams described in proposition 3 admit no mutations.

Note that in type 1 of proposition 3 , we have the $(2, q)$-torus knot diagram as $P(1, q)$. For $k=2$, type 2 a gives the arborescent diagram $(l, l)(-l,-l)$, and type $2 \mathrm{~b}$ the rational diagram $(2 l,-2 l)$. Types 1 and 3 give alternating odd crossing number 
examples, while types 2 and 4 yield non-alternating diagrams of even crossing numbers except those of the form $2 \cdot 3^{l}$. Note also that all the diagrams are positive. Let us in relation to this remark the following.

Lemma 5. Positive or alternating diagrams D are not SEE (unless all crossings are nugatory).

Proof. When $D$ is alternating, then $D$ and $D^{\prime}$ can be distinguished by the using lemma 2 , or alternatively by span $V$ (see [Th]).

If $D$ is positive, then such diagrams can be distinguished using the Vassiliev invariants $v_{2}$ and $v_{3}$ of degree 2 and 3 , as noticed in lemma 4 .

We designed a fast yet efficient computational test for everywhere equivalence based on comparing the degree-2 Vassiliev invariants of all $D^{\prime}$. It enabled us to verify:

(a) prime diagrams up to 18 crossings, and

(b) prime diagrams of genus up to $2 / 3 / 4$ with up to $40 / 30 / 25$ crossings.

Our partial verification must be nevertheless held as rather unrepresentative for the general case. For example, the last two types of links in proposition 3 are far beyond the range of our experimental observation. For type 3 the simplest diagram (46), coming from $P(3,3)$ and $q=3$, has 27 crossings and genus 10 . (See the left diagram in example 1 in $\S 5$, for $P(3,2)$ and $q=3$, which gives a link.) The first essential instance of type 4 , obtained by applying (47) for $P(3,2)$ on $\tilde{Q}(2,2)$, has 24 crossings and genus 9 . (Compare with the middle diagram in figure 9 for $\tilde{Q}(3,2)$.)

Still, everywhere we looked the (first two) patterns in proposition 3 exhausted all examples in which not both $D$ and $D^{\prime}$ are unknotted. In particular, the prediction that there is not a single such 18 crossing (prime) diagram is indeed true. (Whether one occurs for 54 crossings is a mysterious riddle.) The outcome also means that (within the range of our verification) the case that $D^{\prime}$ is knotted, but $D$ is not, never occurs, and that in the opposite case $D$ knotted, $D^{\prime}$ not, the list (44) has no visible succession. This lends some curiosity to the following extension of the related problem in $[\mathrm{SA}]$.

Question 1. Assume $D$ is a prime everywhere equivalent diagram.

1. If $D$ is SEE, is $D$ (and $D^{\prime}$ ) unknotted?

2. If $D$ is WEE, is then $D$ one of the diagrams in (44) or proposition 3 ?

3. If $D^{\prime}$ is knotted, is $D$ always positive?

Note that part 3 is a special case of the combination of parts 1 and 2 .

Let us make a remark on composite diagrams. If a composite diagram $D=\#_{i} D_{i}$ is SEE, then all $D_{i}$ are SEE, and so, expectably, $D_{i}$ are unknotted. We formulate the following property when $D=\#_{i} D_{i}$ is WEE.

Lemma 6. If the answer to question 1 is affirmative and $D=\#_{i} D_{i}$ is WEE, then all $D_{i}$ are among the same of the below three types:

(a) positive WEE diagrams in proposition 3,

(b) their (negative) mirror images, or

(c) among the four figure-8-knot diagrams in (44).

Proof. If $D=\#_{i} D_{i}$ is WEE, then all $D_{i}$ are WEE. Thus if the answer to question 1 is affirmative, then $D_{i}$ are among the listed three types. We thus need to argue that two different types cannot occur.

First assume that a figure-8-knot diagram $D_{1}$ occurs with some factor $D_{2}$ of another type (and possible further factors). 
Note that $D_{2}^{\prime}$ always depicts a positive or negative (or the trivial) knot. A positive or negative knot has no figure- 8 knot factor, and thus $D_{1}^{\prime} \# D_{2} \# \ldots$ and $D_{1} \# D_{2}^{\prime} \ldots$ will have a different number of figure- 8 knot factors.

Thus we remain to rule out a positive factor $D_{1}$ and a negative factor $D_{2}$.

Now, whenever $D$ is a WEE diagram in proposition $3, D^{\prime}$ either simplifies to an unknot diagram (if $D$ is one of the few trefoil diagrams in (44)), or it simplifies to a positive prime diagram of two fewer crossings than $D$. By the result in $[\mathrm{O}]$, both $D$ and $D^{\prime}$ represent prime knots (unless $D^{\prime}$ represents the unknot).

Moreover positive (non-trivial) knots are not negative. Thus looking at the positive and negative prime factors of $D_{1}^{\prime} \# D_{2} \# \ldots$ and $D_{1} \# D_{2}^{\prime} \ldots$, we see that $D_{i}^{\prime}$ and $D_{i}$ must have the same knot type, which is not the case (e.g., by lemma 4).

In fact, one can likely, stronger than the statement of the lemma, conclude that the knot types of all $D_{i}$ are the same. This basically requires to verify that for the positive diagrams $D$ in proposition 3 , all the apparently distinct knot types of $D$ and $D^{\prime}$ are indeed distinct. We have not done all (and so omit here) the technicalities. The (weaker) statement in the lemma will be needed later, though, and thus we wrote an argument with some care.

A refinement of our computational test led to the following partial confirmation results for low genus diagrams.

Theorem 5. Let $D$ be a prime EE diagram of genus up to 4 . Then the answer to question 1 is affirmative if $D$ has one of the following properties: SEE, positive, alternating, or genus at most 3 . Precisely:

1. If $D$ is SEE, then $D$ is unknotted.

2. If $D$ is positive, then $D$ is among the diagrams of proposition 3 , namely $\hat{P}(p, q)$ for $3 \leq q \leq 9$ odd and $\tilde{Q}(k, l)$ for $2 \leq k \leq 5$ and $l \geq 1$, with $l$ even when $k=3$.

3. If $D$ is alternating, then $D$ is a pretzel diagram $\hat{P}(p, q)$ for $3 \leq q \leq 9$, or the 4-crossing figure- 8 knot diagram.

4. If $D$ has genus at most 3 and is WEE, then it is among the diagrams in (44), or a pretzel diagram $\hat{P}(p, q)$ for $3 \leq q \leq 7$, or $\tilde{Q}(k, l)$ for $2 \leq k \leq 4$ and $l \geq 1$, with $l$ even when $k=3$.

Note that in the second part, positivity is assumed, and not (as question 1 suggests) claimed. We do not yet feel confident about treating general WEE diagrams of genus 4 . The reason will become apparent in the proof of theorem 5 . It requires some discussion, and thus occupies the whole next subsection.

4.4. Proof of Theorem 5. All four parts are proved essentially along the same pattern.

Our outset will be that for a potentially EE diagram $D$, we look at the corresponding generator $\hat{D}$ with classes $1, \ldots, g$. (Thus $g$, as in $\S 2.9$, and unlike elsewhere, denotes the number of classes of $\hat{D}$.) After seeing what one has to do with unsigned classes, depending on the situation, we will assume $D=\hat{D}_{y}$ determined by a twist vector $y$.

Let $D_{i}^{\prime}$ be the diagram obtained from $D$ by switching a crossing in class $i$. We then examine conditions of the sort

$$
v_{n}(D)=v_{n}\left(D_{i}^{\prime}\right)\left(\text { for SEE) resp. } v_{n}\left(D_{i}^{\prime}\right)=v_{n}\left(D_{j}^{\prime}\right)(\text { for } \mathrm{WEE})\right.
$$

for Vassiliev invariants $v_{n}$ as constraints on the twist vector $y$ of $D$ (for $n=2,3,4,5$, as far as we need). It is a consequence of the work in [St2] (already invoked in the 
proof of theorem 2) that equalities like (48) give algebraic conditions of degree (up to) $n-1$ on that vector. Thus first, for $n=2$, we restrict possible $y$ to a certain (affine sub)lattice of $\mathbb{Z}^{g}$. In many cases this lattice is already empty (the solutions over $\mathbb{Q}$ of (48) give an affine space with no integer points). If it is not, we examine on it the vanishing of the higher order polynomials, until we are left only with (twist vectors giving) diagrams we claimed. Several extra restrictions on $y$, depending on the type of diagrams we examine, will help us along the way.

We always start with a finite, but rather large, number of generators ( $c f$. table (14)). First we perform the simpler tests using MATHEMATICA, which narrows the list of worthwhile cases. We will explain for the proof of each of the four statements of the theorem what was ordered to the program, and what was the outcome. The remaining cases are examined by hand on an individual basis. This list is still too long (and of little interest) to be presented in entirety, but we give a few representative, and among the more subtle, examples to show how we proceeded.

The four parts of the theorem are ordered chronologically, and essentially with increasing complexity of the test procedure. Thus the balance shifts between what had to be done by computer, and what was left to manual records. For a general WEE diagram of genus 4 , some even more delicate programming will be needed, and/or the ad hoc treatment of an exceedingly daunting remaining list. Thus, even although the result would be more general and less technical, we felt that the effort for the proof grows disproportionally to the importance of the statement.

Part 1. Consider first the claim on SEE diagrams.

Working recursively over crossing number, we argue first that it is enough to consider prime diagrams $D$ without a trivial clasp up to flypes, in particular such with no unsigned classes.

For SEE diagrams with unsigned classes the inductive argument over the crossing number works thus: Let $D^{*}$ be the diagram obtained from $D$ after flypes and resolving a trivial clasp. (Note that $D^{*}$ can be composite even if $D$ is prime.) Flypes commute with crossing changes and do not affect the SEE property. Similarly neither does resolving (though not creating) a trivial clasp. Thus, as remarked above lemma 6 , $D^{*}$ is SEE (even if composite). Eliminating a trivial clasp in an SEE diagram, and decomposing the result into prime factors, does not increase the diagram genus, and gives SEE diagram(s) of fewer crossings. By induction every prime factor of $D^{*}$ is unknotted. The connected sum of unknotted diagrams is unknotted, and then so is $D$.

Thus, until the end of the proof of part 1, we need to consider only prime diagrams $D$ with no unsigned classes. Then we can work with twist vectors. Every component $y_{i}$ of the twist vector corresponds to a class $i$ of the generator. We number the classes $i=1, \ldots, g$. The order is not really relevant, but was chosen in the following calculation by the first crossing in the notation of Dowker and Thistlethwaite [DT], in which generators are maintained. The restriction remains that $y_{i} \neq 0$ when the $i$-th class is even, and this condition will be used extensively.

Now when $D$ is in the series of $\hat{D}$, we examine $v_{2}(D)-v_{2}\left(D^{\prime}\right)$, where $v_{2}$ is the Vassiliev invariant of degree 2. The difference is given by the linking number of the 2-component link obtained by smoothing the switched crossing $p$. This depends only on the $\sim$-equivalence class of $p$.

Let for $D=\hat{D}_{y}$, consider the diagram $D^{\prime}=D_{i}^{\prime}$ be obtained from $D$ by switching a crossing in class $i$. Unless $y_{i}=0$ and the class is even, one can then, after possible flypes, remove a trivial clasp. We assume we do so, and thus always regard $D_{i}^{\prime}$ as 
one of $\hat{D}_{y \pm e_{i}}$, for the standard basis vector $e_{i}$ of $\mathbb{Z}^{g}$ (with 1 in the $i$-th position, and 0 everywhere else). The sign \pm is opposite to $\operatorname{sgn}\left(w_{i}\right)$ (or either signs are possible if $\left.w_{i}=0\right)$. However, the sign is not relevant, because

$$
v_{2}\left(\hat{D}_{y+e_{i}}\right)-v_{2}\left(\hat{D}_{y}\right)=v_{2}\left(\hat{D}_{y}\right)-v_{2}\left(\hat{D}_{y-e_{i}}\right)
$$

and so the vanishing on this expression does not depend on the sign of the crossing we switch.

Now $v_{2}(D)-v_{2}\left(D^{\prime}\right)$ is the sum of the writhes of the $\sim$-equivalence classes linked with class $i$. We obtain then from

$$
v_{2}(D)-v_{2}\left(D_{i}^{\prime}\right)=0 \text { for all } i=1, \ldots, g
$$

a linear $g \times g$ equation system

$$
A_{\hat{D}} y=b,
$$

where the matrix $A=A_{\hat{D}}$ is given by

$$
A_{i j}= \begin{cases}1 & \text { if class } i \text { and } j \text { are linked, } \\ 0 & \text { if class } i \text { and } j \text { are not linked, }\end{cases}
$$

and the vector $b$ by

$$
b_{i}=\frac{1}{2} \#\{\text { odd classes linked with class } i\}
$$

(Note that $b_{i}$ are integers, essentially because of the even valence property.) Solving this system gives an affine subspace $L=L_{\hat{D}}$ of $\mathbb{Q}^{g}$ in which all $y$ lie where $D=\hat{D}_{y}$ is "strongly everywhere $v_{2}$-equivalent", i.e. satisfies condition (50).

We calculated these affine spaces $L_{\hat{D}}$ for all generators $\hat{D}$ using MATHEMATICA. Still $y$ must be in $\mathbb{Z}^{g}$, and many of these affine spaces contain no integer points. Moreover, we need that $y_{i} \neq 0$ when $\# i=2$; otherwise we have a diagram $D$ with a trivial clasp. These restrictions turn out severe: computation shows that such integer points in $L_{\hat{D}}$ occur only for 2 generators of genus 3 (out of 4017 ; cf. table (14)) and 31 of genus 4 (among the nearly 3.5 million).

Now $A$ is a square matrix, but it may sure have a corank $r$. (The largest corank we had was 5.) Then the relevant twist vectors $y$ are of the form

$$
y=a+\sum_{k=1}^{r} c_{k} b_{k}
$$

where $a$ is one element of $L_{\hat{D}}$ and $\left(b_{k}\right)$ is a basis of the kernel of $A_{\hat{D}}$. Note that even if a basis $\left(b_{k}\right)$ is integral, not necessarily for every integer point $y$ the $c_{k}$ must be integers. To ascertain this, we had to manually readjust the bases for two generators $\hat{D}$.

From now on we can work with twist vectors $y$ and their diagrams $D=\hat{D}_{y}$, parametrized by integers $c_{k}$ through (52). The next step is to do a similar test using $v_{3}$ of (11).

Consider therefore the difference

$$
v_{3}(D)-v_{3}\left(D_{i}^{\prime}\right),
$$


for a positive class $i$ of $D$. In case of $v_{2}$ this was a linear function in the $c_{k}$; now it is a quadratic polynomial. (We mentioned that for a Vassiliev invariant of degree $n$ this difference is a polynomial of degree at most $n-1$.)

Note that the difference (53) for the given choice of $D_{i}^{\prime}$ would be relevant only if $w_{i} \geq 0$. For the opposite sign of crossing to switch we would have a separate polynomial. However it turns out that when the expression in (49) is zero, the analogue of (49) holds also for $v_{2}$ replaced by $v_{3}$ :

$$
v_{3}\left(\hat{D}_{y+e_{i}}\right)-v_{3}\left(\hat{D}_{y}\right)=v_{3}\left(\hat{D}_{y}\right)-v_{3}\left(\hat{D}_{y-e_{i}}\right) .
$$

The property (54) can be deduced from the identity

$$
V\left(\hat{D}_{y+e_{i}}\right)=\left(t^{2}+1\right) V\left(\hat{D}_{y}\right)-t^{2} V\left(\hat{D}_{y-e_{i}}\right),
$$

which in turn follows from (1) (and was used e.g. in [St6]), and the expressions of $v_{2}$ and $v_{3}$ in terms of $V^{\prime \prime}(1)$ and $V^{\prime \prime \prime}(1)$ (see (11) and above it). With (54), the vanishing of (53) can thus again be assumed not to depend on the sign of class $i$.

Regarding the difference (53) as expressed in terms of $c_{k}$, we replace now (51) by a quadratic equation system in $c_{k}$, with $r$ variables and $g$ equations. Despite that $r<g$, this system is often underdetermined; sometimes even all equations are trivial. However, many non-trivial quadratic polynomials are simple; often they are single monomials. Their vanishing then means that some $c_{k}$ is zero. It turns out then that this implies via (52) that $y_{i}=0$ for some $i$ with $\# i=2$. Then $D$ has a trivial clasp (up to flypes), a case we excluded, though.

This leaves now a few very specific diagrams. Many of them are directly checked to be unknotted. Then we remain at 5 generators. With them a similar test using $v_{4}$, the coefficient of $z^{4}$ in the Conway polynomial, works. (Note that $v_{4}$ also satisfies a property like (49) and (54), which follows from (10).)

This procedure finishes the proof for the SEE case.

Part 2. The method can, in principle, be used also for WEE diagrams. Then, though, the $v_{2}$-test means that a twist vector $y$ with WEE $\hat{D}_{y}$ must satisfy an equation system obtained from (51) by multiplying the $i$-th equation by $\operatorname{sgn}\left(w_{i} w_{1}\right)$ (see (15)), and then subtracting the first equation of the system (which is discarded) from all others. Let this new system be denoted as

$$
\tilde{A}_{\hat{D}} y=\tilde{b} .
$$

This system depends on the signs of $w_{i}$, up to reversing all of them simultaneously. Thus we have to deal not with one, but with $2^{g-1}$ systems for each generator $\hat{D}$, which is too tedious (at least for genus 4; see the remark before the proof).

However, if we focus on positive (prime) diagrams, we have again one equation system per generator. The rank of $\tilde{A}_{\hat{D}}$ often decreases in comparison to $A_{\hat{D}}$, and there are many more $\hat{D}$ with integer points in the solution space $\tilde{L}_{\hat{D}}$ of $(55)$. However, now a new condition enters for the twist vector $y$ : all $y_{i}$ (or equivalently, $w_{i}$ ) are positive.

This again brings the relevant generators $\hat{D}$ down to a manually manageable list. We can then perform an analogous test with $v_{3}$ and $v_{4}$. Let

$$
P_{i j ; n}=v_{n}\left(D_{i}^{\prime}\right)-v_{n}\left(D_{j}^{\prime}\right) .
$$

Now we have to examine the vanishing of quadratic polynomials $P_{i j ; 3}$ in the coefficients $c_{k}$ of (52) (or simpler, it is enough to look just at $P_{1 j ; 3}$ ) and, when needed, the cubic polynomials $P_{i j ; 4}$. To give an idea how this goes, we include a specific example. 
The 24 crossing generator, given in the notation of Dowker and Thistlethwaite $[\mathrm{DT}]$

64210481441882412403016463422382844322023626

has the following solution of (55), where we order the 12 classes by the first crossing occurring in the above notation. The vector $a$ in (52) is the zero vector (so $L_{\hat{D}}$ is here a genuine linear space), and $r=3$ with

$$
\begin{aligned}
& b_{1}=(1,1,1,1,1,1,0,1,0,1,1,1) \\
& b_{2}=(-1,0,0,1,0,-1,0,0,1,0,0,0) \\
& b_{3}=(0,-1,1,0,-1,0,1,0,0,0,0,0)
\end{aligned}
$$

By looking at coordinates 7, 9 and 10, we see that in order $y$ in (52) to be an integer vector, all $c_{k}$ must be integers.

It turns out that the $v_{3}$-test gives no new restriction on this linear space, i.e., $P_{i j ; 3}=0$ for all $i, j$. (Note that, while the equality (54) may no longer hold, we know that we always have to look at the term on its right hand side. Now this term is not supposed to vanish, but to be equal for all $i$.)

Looking at $v_{4}$, let $D_{i}^{\prime}$ be again the diagram obtained from (a positive diagram) $D$ by switching a crossing in class $i$ (where $i=1, \ldots, 12$ ). By calculating $P_{13 ; 4}$ of $(56)$ in a few explicit diagrams, we find

$$
P_{13 ; 4}=2 c_{2} c_{3}\left(c_{2}+c_{3}\right)+2 c_{1} c_{3}\left(c_{3}-c_{1}\right)-2 c_{1} c_{2}\left(c_{1}+c_{2}\right) .
$$

Now observe that the positivity of $y_{i}$ imposes the restrictions $c_{1}>c_{2}, c_{3}>0$ on $c_{k}$. It is then, however, easy to see that $P_{13 ; 4}<0$ for $c_{k}$ satisfying these inequalities. Thus none of these positive diagrams $D$ is everywhere equivalent.

Part 3. It was already indicated in part 2 how to handle diagrams where classes have crossings of given sign. We tried this out in the, most interesting, case of alternating diagrams. For a negative (crossing) class $i$ the restriction on the twist vector component $y_{i}$ is now $y_{i} \leq 1-\# i$.

We restricted ourselves only to non-special generators, since for the special ones the properties positive and alternating are equivalent, and thus we are back to part 2 .

Testing for integer points in the solution of (55) and (in some rudimentary and not thorough way) the admissibility of the proper signs of $w_{i}$, we had 121 generators of genus up to 4 . We used on them the $v_{3}$ test. (Again (54) may no longer hold, and we must choose what hand side to look at depending on $\operatorname{sgn}\left(w_{i}\right)$.)

We combined the $v_{3}$ test with the following extra argument. We have observed (e.g. in [St7]) that a $\bar{t}_{2}^{\prime}$ move in a positive class of an alternating diagram increases $\max \operatorname{deg} V$ by 2 , and fixes min $\operatorname{deg} V$. In a negative class, it decreases min $\operatorname{deg} V$ by 2 and fixes max $\operatorname{deg} V$. This implies that no alternating diagram $D$ can be EE when

$$
\text { there are some } 1 \leq i, j \leq g \text { with } w_{i},-w_{j} \geq 3 \text {. }
$$

We illustrate the argument by the following example. For the 17 crossing generator

481822220263061234281432162410 
we have 13 classes, only the sixth of which is negative in an alternating diagram. The linear system (55) has the affine (integer) basis, with $r=3$,

$$
\begin{aligned}
& a=(-1,0,1,0,0,1,0,0,-1,0,0,0,0), \\
& b_{1}=(-1,0,1,0,0,0,0,-1,-1,1,0,0,1) \text {, } \\
& b_{2}=(4,1,-3,1,1,-2,1, \quad 0, \quad 4,1,0,1,0) \text {, } \\
& b_{3}=(0,0,0,0,0,0,-1,1,0,-1,1,0,0) \text {. }
\end{aligned}
$$

From the condition $y_{j}>0$ when $j=11,12,13$, we see that $c_{i}>0$ for $i=1,2,3$. Now look at the sixth coordinate in (52). If $c_{2}>1$, then we find $y_{6}=1-2 c_{2} \leq-3$, while $y_{12}=c_{2}>1$. Thus $w_{6} \leq-6$ and $w_{12} \geq 3$, which cases can be excluded by the test (57).

Thus $c_{2}=1$. Now, though, looking at the seventh coordinate, we have $y_{7}=$ $c_{2}-c_{3}=1-c_{3} \leq 0$, in contradiction to $w_{7}>0$, that is needed for a positive class.

The vanishing test of $P_{i j ; 3}$, together with the test (57) and the condition on the signs of $w_{i}$, allows us to deal with all the remaining generators in a way similar to the above example. We are finally left only with the 4-crossing (figure-8-knot) diagram.

We emphasize the importance of the test (57) as follows. In some cases, the polynomials $P_{i j ; n}$ (for $n=3,4$ ) of $(56)$ were too complicated to easily give restrictions on the $c_{k}$. In other cases they were too hard to obtain. Our approach to determining $P_{i j ; 3}$ (and $P_{i j ; 4}$ ) was via diagrammatic calculation of $v_{3}$ (resp. $v_{4}$ ). Namely, since we know the maximal possible degree of $P_{i j ; n}$, the polynomial can be determined from its values on (absolutely) small arguments $c_{k}$. These determine via (52) particular diagrams $\hat{D}_{y}$. However, the bases $\left(b_{k}\right)$ of the lattices we obtained for some genus 4 generators were not simple, in that they had large coordinates (in absolute value; the largest one we had is 57). Thus even small $c_{k}$ can lead to large $\left|y_{i}\right|$ and diagrams with many hundred crossings, on which $v_{3}$ would have to be evaluated. The test (57) made it redundant to treat such complicated cases. The unavailability of (57) to avoid these situations is one main reason for the considerable increase in difficulty of the test for non-alternating diagrams.

Part 4. We have tried, in the end, to look at the full set of systems (55) at least for genus up to 3 . We explained that they depend on the pair $\left(\hat{D},\left(\operatorname{sgn}\left(w_{i}\right)\right)_{i=1}^{g}\right)$, which we will call a signed generator. Before we describe the handling of signed generators, we discuss, though, another additional case which returns to relevance: the diagrams $D$ with unsigned classes.

Case 1. Unsigned classes do not occur for positive or alternating diagrams, and for SEE diagrams the inductive argument over the crossing number worked easily. We wrote lemma 6 mainly in order to see here how to deal with the WEE case.

Now, if $D$ is WEE, $D^{*}$ is also. (We use $D^{*}$ with the meaning of part 1.) Note that a WEE diagram has no nugatory crossings. We use again an induction argument over crossing number and want to apply lemma 6 . This is justified under induction assumption by repeating the argument we had for SEE that the diagram genus does not increase under flypes and clasp resolution.

Lemma 6 then says that either $D^{*}$ is positive, or a possible connected sum of the figure-8-knot diagrams in (44). The case that $D^{*}$ is positive is handled by the following lemma.

Lemma 7. A diagram D with exactly one negative crossing is not EE.

Proof. One of the $D^{\prime}$ will be positive, while others will be not. This is the situation treated in lemma 4. 
However, the case that $D^{*}$ is a connected sum of some of the four right (figure8) diagrams in (44) must be paid attention to. The genus does not increase after resolving a clasp, and by checking the genus of the diagrams in (44), we see that a diagram $D^{*}$ of genus 3 can have at most 15 crossings (the connected sum of 3 of the 5 crossing diagrams). So $D$ has at most 17 crossings. This already leaves a finite range of diagrams, but we mention two arguments which further considerably narrow this range, and make the check practicable.

First, if an unsigned class has more than two crossings, then $D^{*}$ is prime (after removing the trivial clasp). It is then one of the diagrams in (44) (without connected sum). In these diagrams all crossing switches give the unknot. To show that $D$ is not EE, it is enough to check that a $\bar{t}_{2}^{\prime}$ move at whatever crossing of a diagram in (44) gives no unknot. This can be seen using $v_{2}$ without electronic intervention: the invariant of these diagrams will be -2 , since the diagram $D$ depicts figure- 8 with $v_{2}=-1$, and the unknot has zero invariant.

We then have to deal only with the case that unsigned classes have two crossings. We can exclude next the diagrams with more than one such class. Namely, in this case $D^{*}$ would still have an unsigned class. This can then be ruled out by induction, since our list of WEE diagrams has none with an unsigned class.

Thus we need to check diagrams $D$ of at most 17 crossings, with exactly one unsigned class, and this class having two crossings. These diagrams were examined by a case-by-case (computer) verification.

Case 2. We can now turn to diagrams with signed classes only. One can assume up to mirroring (and we did) that $\operatorname{sgn}\left(w_{1}\right)=1$. After about 10 hours of computation, verifying the solvability of the systems $(55)$, MATHEMATICA leaves $\approx 1200$ signed generators for further treatment.

At this point, not only the determination (as in the previous parts of the proof), but also most of the test of $P_{i j ; 3}$ had to be automatized. Recall that $P_{i j ; 3}$ are quadratic polynomials in the $c_{k}$. It turns out in practice that very often they depend on only one variable among the $c_{k}$. In that case, the roots can be easily found, and they must be rational (or often even integral, when the integrality of $c_{k}$ can be deduced from the integrality of $y$ via (52)). Whenever they are not, the signed generator can be discarded.

We also discarded signed generators which correspond to positive or alternating diagrams, since these were treated in the previous two parts of the proof. We thus aim to remain only with the figure-8-knot diagrams in (44) (except the alternating one).

With this we reduced the list of signed generators from about 1200 to about 200 . For them $P_{i j ; 3}$ can be examined closer. Here we left it to MATHEMATICA to solve the system of polynomial equations

$$
\left(P_{i j ; 3}=0\right)_{i, j}
$$

in $\left(c_{k}\right)$. (Again, it is enough to take $i=1$.) In the previous parts of the proof we had tried to rule out or restrict integer solutions to (58) by looking at the polynomials manually, in particular because we were not confident that MATHEMATICA's output would be manageable. But here the program performed unexpectedly well, and revealed that for most generators these systems are unsolvable. In a few cases, it yielded sporadic solutions. These, however, are not real or not rational or integral, or do not lead to the proper signs of $w_{i}$.

Apart from the three non-alternating figure-8-knot diagrams in (44), we were left only with two signed generators for which (relevant) solutions of (58) occur. 
These arise, for certain signs of classes, from the 16 crossing generator $\tilde{Q}(4,2)$ (made alternating by crossing switches). The $v_{4}$ test reduced the two parameters of each family to one, and that one had to be eliminated using the degree-5 Vassiliev invariant $v_{5}$ of $(12)$.

5. A graph-theoretic construction. Finally, we discuss a relation to graph theory, as outlined in the introduction. We admit (responding to advice by the referee), that this relation occurred to us clearly only after we had observed and examined (on low genus diagrams) the knot theoretic constructions in the previous section. We convinced ourselves in the end (see propositions 4 and 5) that for knots the below graph-theoretic point of view only reformulates, and does not extend, the families of everywhere equivalent diagrams we obtained. However, it gives a way of observing the generalization to link diagrams, showing a natural relationship to totally symmetric graphs. In the belief that studying this relationship might be worthwhile, we present $\S 5$ as a conclusion of the paper.

5.1. Edge transitive graphs. We recall the generalities regarding graphs put together in $\S 2.4$.

As mentioned, we will consider everywhere equivalence below also for link diagrams. For our purpose we should assume that all $D^{\prime}$ represent unorientedly isotopic links. Isotopy must be understood also to allow interchange between components of $D^{\prime}$. It should still, though, preserve mirroring. For everywhere equivalent oriented link diagrams, isotopy may both alter orientation of individual components and interchange some of them.

Throughout the below exposition, $G$ will play the role of a checkerboard graph $G=G(D)$ of a diagram $D$.

Let us in the following restrict ourselves to prime diagrams $D$. (We discussed how to infer about composite ones in and above lemma 6.) Consequently, we will assume below that $G$ is cut-free. Note also that a cut-free graph $G$ is 2-connected (has no isthmus), unless $G$ is a single edge. Here is a way to generate some families of everywhere equivalent diagrams.

Let $P(p, q)$ be a pretzel tangle as in $(45)$, and let $P(1, q)$ be called a $q$-twist. For a graph $G$ with $G=G(D)$, we define diagrams $D_{1}(G ; q)$ resp. $D_{2}(G ; q)$ by replacing each edge $e$ of $G$ by a $q$-twist in edge direction, resp. a twist opposite to edge direction as follows (for $q=5$ ):

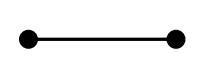

edge in $G$

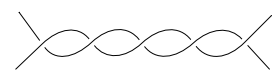

twist in edge direction

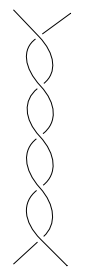

twist opposite to edge direction

For $p>1$, we can define $D_{1}(G ; p, q)$ and $D_{2}(G ; p, q)$ analogously, when setting the direction of the pretzel tangle so that for $p=1$ we obtain the previous picture. (Thus, for example, $P(3,5)$, as positioned in (45), should be parallel to a horizontal edge $e$ as in (59).)

That is, $G\left(D_{1}(G ; p, q)\right)$ is obtained from $G$ by bisecting $(q-1)$ times each edge of $G$, and doubling $(p-1)$ times each of the resulting $q$ edges. (For $G\left(D_{2}(G ; p, q)\right)$, double $(q-1)$ times, and then bisect $(p-1)$ times.) 
Thus,

$$
D_{1}(G ; p, q)=! D_{2}\left(G^{*} ; p, q\right) .
$$

In (59), the twist in edge direction is Kauffman negative (and in the checkerboard coloring, the bigons are colored black), while twist in opposite to edge direction are Kauffman positive (and the bigons they create are colored white).

Construction 1. Let $G$ be a cut-free edge transitive graph and fix $p=1,3$ and $q \geq 1$. Consider the link diagram $D_{i}=D_{i}(G ; p, q)$ for $i=1,2$. That is, replace each edge $e$ of $G$ by a $P(p, q)$-pretzel tangle either along, or opposite to, the direction of $e$. Choose crossings of $D_{i}$ to be all Kauffman positive.

When $G$ has a reflection symmetry that reflects an edge (i.e., exchanges its endpoints), then consider also $D_{1}(G ; p, 2)$ for $p \geq 1$. (We will call this the reflective case.)

EXAmPle 1. Let $G=M_{3}$ be the theta-curve, $p=3$ and $q=2$. The two diagrams are shown below. They are manifestly different: among others, the first one has 3 components, the second one two.

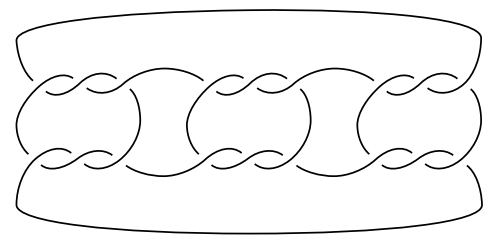

$D_{1}\left(M_{3} ; 3,2\right)$

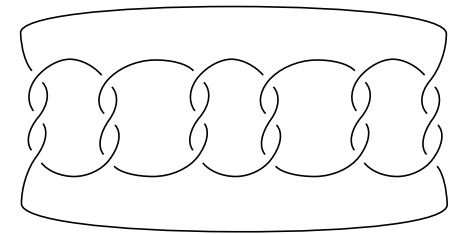

$D_{2}\left(M_{3} ; 3,2\right)$

It is possible to take also $p=2$, but then we must either demand that $q=2$ and (depending on the direction of the twists) that $G$ is vertex or face transitive, or we must regard $D^{\prime}$ as equivalent also under changing ways to build connected sum.

Remark 1. Note that when $G$ has an edge-reflecting symmetry, $G^{*}$ has an edge-fixing one. Both types need to be separated here. For example, let $G$ be the cuboctahedron, the median graph of the cube net, or the icosidodecahedron, the median graph of the dodecahedron net. Then both $G$ and $G^{*}$ are edge-transitive (see the proof of proposition 4 below). Moreover, $G$ has an edge-reflecting symmetry, and thus $G^{*}$ has an edge-fixing one, but not vice versa. Thus $D_{1}(G ; p, 2)$ is EE, but not necessarily $D_{1}\left(G^{*} ; p, 2\right)=! D_{2}(G ; p, 2)$.

By lemma 3 , one can characterize those $G$ giving a knot diagram $D$ in construction 1 by saying that $G$ should have odd number of spanning trees. Note the following important consequence of this property: whether a graph $G$ gives a knot diagram $D$ with $G=G(D)$ does not depend on the planar embedding of $G$. We will not refer to this circumstance explicitly, but to follow some of the below arguments, it will be helpful to choose a favorable embedding of $G$ to decide whether it gives a knot diagram.

Lemma 8. If $D$ is obtained by construction 1 , then $D$ is everywhere equivalent. Moreover, $D$ is alternating and, if it is a knot diagram, it is (up to mirroring) positive, i.e., special alternating. 
Proof. Since $D$ is constructed from $G$, the symmetry of $G$ that carries $e$ to $e^{\prime}$ will carry the twists of $e$ in $D$ to those of $e^{\prime}$. If the plane homeomorphism alters the orientation, then it is realized by a $\pi$-rotation of the link in space, which mirrors the diagram and then changes all crossings. Thus in these cases again Kauffman positive twists are carried to Kauffman positive ones.

When all crossings are Kauffman positive (or all are Kauffman negative), then $D$ is alternating. It remains to see that for a knot diagram, all crossings are either (skein) positive or all are (skein) negative. For this it is enough to look at $p=1$ (instead of $p=3$ ), where $D$ consists of $q$-twists. The reflective case gives no knot diagrams; see the proof of proposition 4 below.

The sign of Kauffman positive twists depends on the local orientation of the strands: Kauffman negative reverse twists are (skein) positive, while Kauffman negative parallel twists are (skein) negative (opposite with Kauffman positive twists).

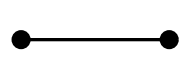

edge $e$

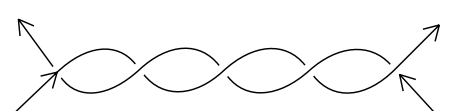

reverse twist

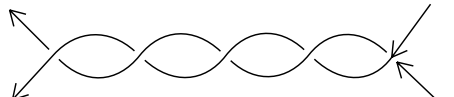

parallel twist

Let $e$ be an edge in $G$ corresponding to a twist in $D=D_{i}(G ; q)(i=1,2)$, and let $r$ be a crossing of $D$ in that twist. For every knot diagram $D$ and a crossing $r$, one of the two splicings of $r$ in $D$ as in figure 1, the skein smoothing, gives a 2-component link diagram, and the other one gives a knot diagram. The twist at $e$ is reverse if and only if the skein smoothing of $r$ is the splicing that corresponds (after possible nugatory crossings are removed) to the deletion (as opposed to contraction) $G \backslash e$ of $e$ in $G$.

Now, by lemma 3, this is equivalent to saying that $G \backslash e$ has an odd number of spanning trees. (One can distinguish using the lemma the two spliced diagrams by the parity of this number.) But by symmetry of $G$, this number (and its parity) must be the same for all edges $e$ of $G$. Thus the local orientation of all twists in $D$ is the same, and since (in an alternating diagram) the Kauffman signs of all crossings are the same, so are all skein signs.

The spanning tree number argument given here depends essentially on the property $D$ to be a knot diagram: one cannot in general recognize between an $n$-component and an $(n+1)$-component link diagram for $n>1$ using the number of spanning trees. This is also evident, since altering orientation of individual (diagram) components does not affect the Kauffman sign, but it will alter skein signs of some crossings. Thus one can easily construct a number of counterexamples to the analogous positivity statement for diagrams of several components. For this reason we chose to work only with unoriented everywhere equivalence for links.

Note that families 1 and 3 in proposition 3 arise from construction 1 using the graphs $M_{q}$ of two vertices, where $q$ is odd.

We hoped construction 1 to give more examples in the knot case, but it turns out that it is not so.

Proposition 4. All everywhere equivalent knot diagrams obtained from construction 1 are given by families 1 and 3 in proposition 3.

Proof. Let us put aside the reflective case first (we will come back to it at the end). 
Edge transitive graphs have been studied in combinatorics for some time. If in our case $G$ is 3-connected and dually 3 -connected it gives a planar tesselation. By Whitney's work every symmetry of the abstract graph is realizable by a homeomorphism of the plane (see [CP, p. 2] or [FI, p. 98], for example).

There are only nine finite edge transitive tesselations; see [FI, Theorem 1] and [HS, p. 405]. Five are given by the nets (1-skeletons) of the Platonic solids. The other four graphs are the cuboctahedron and icosidodecahedron (see remark 1), and their planar duals.

It is easily checked that none of the nine graphs gives a knot diagram via construction 1 (i.e., all have even number of spanning trees).

The other cases, we have up to duality a non 3-connected graph. Let us first assume there are no multiple edges. These cases were treated in [FI, Theorem 2], which for convenience we restate here.

THEOREM 6. (Theorem 2 of [FI]) The finite, simple, connected edge-transitive planar graphs which are not 3-connected are the single vertex, the single edge, stars, simple cycles, and the graphs obtained from the single edge, simple cycles and the nets of the Platonic solids, the cuboctahedron, and the icosidodecahedron by replacing every edge by $K_{2, n}$ (the complete bipartite graph), where $n$ is a fixed positive integer.

Replacing an edge $e$ by $K_{2, n}$ means the deletion $e$, followed by the identification of the vertices of valence $n$ in $K_{2, n}$ (or of any pair of nonadjacent vertices for $n=2$ ) with the endpoints of $e$. (E.g., when $n=1$, we have the bisection in (9).)

Note, however, that in the above theorem edge-transitivity w.r.t. symmetries of the abstract graph is considered. When 3-connectivity fails, not all such symmetries come from homeomorphisms of the plane (they come from the flipping phenomena shown by Whitney). In our case a homeomorphism of the plane still preserves the two covalencies $d_{1}$ and $d_{2}$ of each edge (the number of edges bounding the two faces adjacent to the edge). Thus $d_{1}$ and $d_{2}$ are the same (up to order) for each edge.

This argument readily discards most of the graphs in theorem 6 . The star is not 2 -connected (and hence not cut-free). The edge gives the diagrams of family 1 in proposition 3 , and the cycle gives those of family 3 .

The replacement of each edge in a graph $G^{\prime}$ by $K_{2, n}$ will give a graph $G$ whose link diagram has a component (among others) for each face of $G^{\prime}$. Moreover, $\left(d_{1}, d_{2}\right)$ are not equal for all edges of $G$, unless $n=1$ or $G=K_{2, n}$. But $G=K_{2, n}$ gives a knot only if $n=1$, which is of little interest.

It remains to look at the case of multiple edges. By symmetry then all edges are multiple, and then by a recursive nesting argument, some of them must bound a 2 -gonal face. Thus $d_{1}=2$, which means that $G$ is obtained from some graph $G^{\prime}$ by doubling each edge at least once. If some edge is (at least) triple, all must be, and by the same nesting argument we have three multiple edges enclosing two 2 -gons. Then $d_{2}=2$ also, and so we have a two-vertex graph $M_{q}$.

When all edges of $G^{\prime}$ are doubled, then one can still analyze what $G^{\prime}$ is (it must have $d_{1}=2$ ), but it is not necessary. One directly sees that for whatever $G^{\prime}$, construction 1 on $G$ cannot give a knot diagram.

The reflective case is easily seen to give no new knot diagrams. If $p$ is even, then there are always extra components in the tangle $P(p, 2)$. Otherwise, for $p$ odd, the connectivity is the same as for $p=1$, which we already studied.

5.2. Dually edge transitive graphs. The reason for explaining construction 1 is mainly for introducing its analogue which explains types 2 and 4 in proposition 
3, but which is less standard.

Definition 2. Let us call a graph $G$ dually edge transitive if the following conditions are satisfied.

First, $G$ is self-dual, that is, homeomorphic on the plane to its planar dual $G^{*}$. Note that each edge $e \in G$ has its dual edge $e^{*} \in G^{*}$. Thus for each set of edges $E$ of $G$ we have a dual set $E^{*}$ (which is different from the planar dual of $E$ regarded as a graph for itself!).

Let $i=1,2$ and $j=3-i$. We demand that there is a disjoint partition $E(G)=$ $E_{1} \cup E_{2}$ of the edges of $G$ with the following property. If $e, e^{\prime} \in E_{i}$, there should be a symmetry of $G$ taking each of $E_{i}$ to itself and $e$ to $e^{\prime}$. If $e \in E_{i}$ and $e^{\prime} \in E_{j}$, then a symmetry of $G$ should take each of $E_{i}$ to $E_{j}^{*}$ and $e$ to $e^{* *}$.

Even if dual edge transitivity is a tangible property, we are not aware of any attention given previously to it, and of how much graph theoretical interest it is. (See, however, question 2 below and the comment after it.)

EXAMPLE 2. Consider a wheel (graph) $W_{n}$, the result of connecting all vertices of an $n$-cycle graph to an extra, central, vertex (see middle of figure 8 or e.g. [St4]). Further examples of dually edge transitive graphs include the double stars and twofold ${ }^{3}$ wheels. Typical instances of each class are shown in figure 8 . In all three cases, $E_{1}$ can be taken as the set of edges incident from the central vertex. (Thus for double stars, $E_{2}=\varnothing$.)
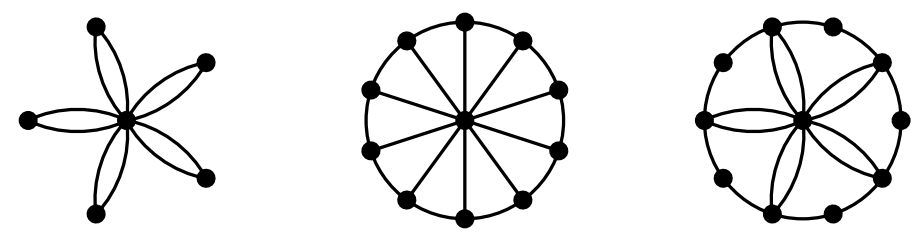

FIG. 8. Dually edge transitive graphs: a double star, a wheel, and a twofold wheel.

Of course, the role of $E_{1}$ and $E_{2}$ in the above definition can be interchanged. This switch will be used (with non-trivial effect), so let us for simplicity write

$$
\bar{E}=E(G) \backslash E
$$

for an edge set $E \subset E(G)$ of $G$. In at least one case (see below example 3) there is an ambiguity of $E_{1}$ beyond taking the complement in $E(G)$. For the following it will thus be helpful to regard a dually edge transitive graph as the pair $\left(G, E_{1}\right)$.

Construction 2 . Let $\left(G, E_{1}\right)$ be a cut-free dually edge transitive graph. Take $p=1,3$ and $q \geq 1$. Replace each edge $e$ of $G$ by a $P(p, q)$-pretzel tangle, oriented in the direction of $e\left(c f\right.$. (59)) if $e \in E_{1}$, and opposite to that direction if $e \in E_{2}$. Switch crossings so that all crossings corresponding to edges in $E_{1}$ (resp. $E_{2}$ ) are Kauffman positive (resp. Kauffman negative). Call the result $D\left(G, E_{1} ; p, q\right)$.

If $G$ has an edge-reflecting symmetry along an edge $e \in E_{1}$, then consider additionally $D\left(G, E_{1} ; p, 2\right)$ for $p \geq 1$ (and again call it the reflective case).

\footnotetext{
${ }^{3}$ We very intentionally use the word 'twofold' here: the twofold wheels are not double wheels, i.e., the doubled graphs of wheel graphs.
} 

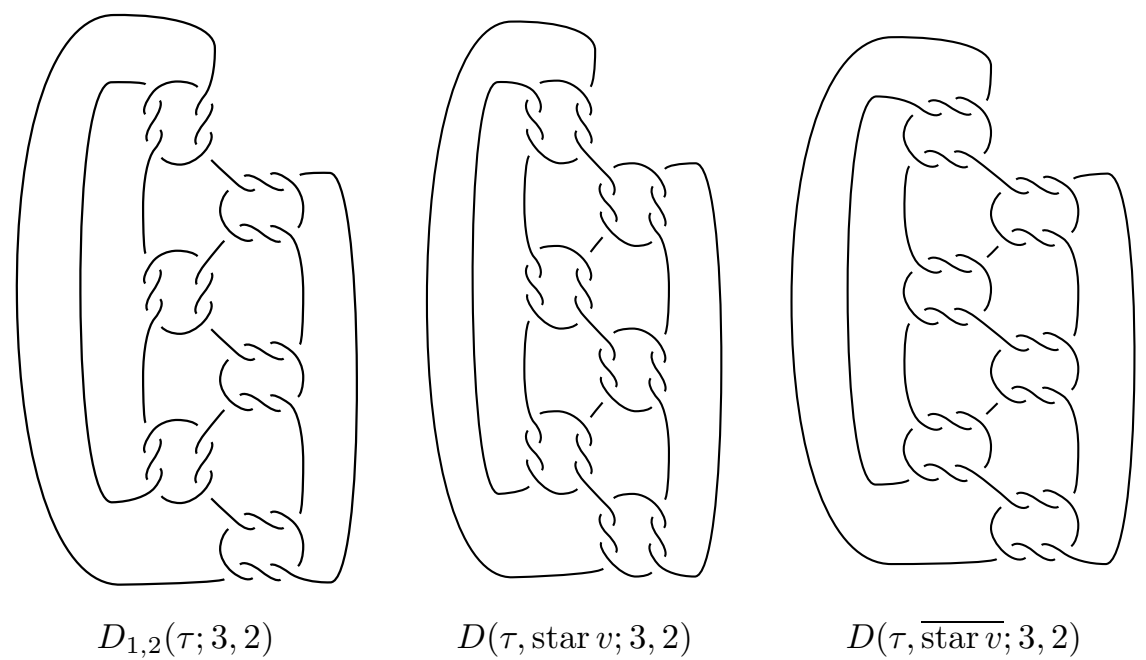

FIG. 9. EE diagrams obtained from $\tau$. In the right two diagrams, $v$ is the vertex of $\tau$ that corresponds to the rightmost bounded region (when the checkerboard coloring is chosen so as this region to be black).

(A similar remark to construction 1 applies in the case $p=2$.)

In order $D$ to be a knot diagram, one can again say that for odd $q$, the graph $G$ should have odd number of spanning trees. For even $q$ one can say that after bisecting all edges in $E_{1}$ and doubling all edges in $E_{2}$ (or vice versa), the number of spanning trees should be odd.

EXAMPLE 3. We show here the case of the tetrahedral graph $G=\tau$, since it has an extra peculiarity. First, $\tau$ is edge-transitive and self-dual, and thus dually edge-transitive with $E_{1}=E(\tau)$ or $E_{1}=\varnothing$. But $\tau=W_{3}$ is also dually edge-transitive as a wheel graph, with $E_{1}=\operatorname{star} v$ (for whatever vertex $v$ ). Below we see several diagrams obtained from $\tau$ by composing with $P(3,2)$.

First, if an edge-transitive graph is self-dual, we have comparing with (60), up to mirroring,

$$
D(G, E(G) ; p, q)=D_{1}(G ; p, q)=! D_{2}(G ; p, q)=! D(G, \varnothing ; p, q) .
$$

Here, for $G=\tau$ and $(p, q)=(3,2)$, we have beside this diagram also $D(\tau, \operatorname{star} v ; 3,2)$ and $D(\tau, \overline{\operatorname{star} v} ; 3,2)$. The three diagrams are shown in figure 9. Apart from having all 36 crossings, they are entirely different: the first is an alternating diagram of 4 components, the other two are non-alternating, of one and 3 components, resp.

REMARK 2. Again, as in remark 1, the reflective case is not duality invariant. Consider the wheel $W_{n}$ with an $n$-valent central vertex $v$, for $n \neq 3$. The graph has edge-reflecting symmetries along edges in the cycle $\overline{\operatorname{star} v}$, but not along those in star $v$ (which connect vertices of different valence). Thus $D\left(W_{n}, \overline{\operatorname{star} v} ; p, 2\right)$ is EE, but not in general $D\left(W_{n}, \operatorname{star} v ; p, 2\right)$.

Lemma 9. A diagram obtained by construction 2 is everywhere equivalent. Moreover, if it is a knot diagram, it is positive. 
Proof. For knot diagrams, an argument similar to construction 1 is needed to ensure the strand orientation at each twist is proper. This goes as before, with the additional remark that switching between $e$ and $e^{*}$ is compensated for by altering the direction of the twists (which changes their local orientation). For knots, the reflective case is again irrelevant (see proposition 5).

Types 2 and 4 in proposition 3 come from construction 2 applied on $G=W_{k}$. In particular, $Q(k, l)=D\left(W_{k}, \overline{\operatorname{star} v} ; 1, l\right)$ for the central vertex $v$, and $\tilde{Q}(k, l)=$ $! D\left(W_{k}, \operatorname{star} v ; 1, l\right)$.

We proposed this second construction, because it raises the following graph theoretic question, which the referee recommended to further highlight:

Question 2. What graphs $G$ are dually edge transitive? Can graphs other than those on figure 8 occur?

Since the double stars are not cut-free, they are not very relevant to us. The twofold wheels, however, were initially unexpected, and came up during our attempt to systematically study the occurring possibilities for $G$. We are quite convinced that the given families are all, but we have not done the complete details to rule out everything else. (However, note for example, that the below proposition 5, together with lemma 3 , implies the case of odd number of spanning trees.)

We have not studied exhaustively question 2 also because graph theory was not our main objective. Coming back to our initial motivation of knot diagrams, we have been able to clarify the most important point in this context, whose proof concludes the paper.

Proposition 5. The knot diagrams yield by construction 2 are families 2 and 4 in proposition 3 (obtained when $G$ is a wheel).

Thus our graph theoretic concept is not helpful in generating more examples of EE knot diagrams. There are, however, some new link diagrams obtained by construction 2 applied on the twofold wheels.

Proof. As for proposition 4, we can easily argue that, once we clarify the occurring knot diagrams outside the reflective case, we see that this case gives nothing new. We will thus not consider the reflective case below.

In the following let $i=1,2$ and $j=3-i$. Let us first stipulate that we understand $E_{i}$ not only as edge sets, but as subgraphs of $G$. The vertices of $E_{i}$ are set to be all vertices of $G$ connected by at least one edge in $E_{i}$ (i.e., we delete isolated vertices). Similarly is done with $E_{i}^{*}$ (which, again, is here not the dual of $E_{i}$ taken for itself).

Now edge transitivity of $E_{i}$ means that $E_{i}$ is a union of some number $n$ of copies of the same connected component $\tilde{E}_{i}$. This graph $\tilde{E}_{i}$ is edge transitive itself, and thus it is either among the graphs in [FI, Theorems 1 and 2], or is obtained from some of these graphs by (possibly iterated) edge doubling. In particular, if some edge of $\tilde{E}_{i}$ is multiple, so are all its edges.

Since each edge transitive symmetry of $E_{i}$ is realizable by a planar homeomorphism, for each $\tilde{E}_{i}$, the same number of its faces contains further $\tilde{E}_{i}$. Now, since the number $n$ of $\tilde{E}_{i}$ is finite, we see that precisely one of the faces of each $\tilde{E}_{i}$ contains further $\tilde{E}_{i}$. We can choose the point at infinity w.l.o.g. so that this is the infinite face.

Next, the number of connected components of $E_{j}^{*}$ is equal to the number of faces of $E_{i}$ containing edges of $E_{j}$. This number must be equal to $n$ (since $E_{i}=E_{j}^{*}$ ). Moreover, by symmetry the same number of faces of each $\tilde{E}_{i}$ contains edges of $E_{j}$. If 
$n>1$, we see that exactly one finite face of each $\tilde{E}_{i}$ contains edges of $E_{j}$, and the infinite face of $E_{i}$ contains none. Now, however, exactly one face (the infinite one) of $E_{j}$ contains edges of $E_{i}$. Thus $E_{i}^{*}=E_{j}$ must be connected, which implies that $n=1$, i.e., $E_{i}=\tilde{E}_{i}$.

Next, edge transitive symmetry implies that for each edge in $E_{i}$ the same number $(0,1$ or 2$)$ of adjacent faces must contain edges of $E_{j}$. This number is obviously not 0 . If it is 2 , connectivity of $E_{j}$ implies that $E_{i}$ has only one face, and no edge can have two adjacent faces. Thus the number is 1 . Since we argued that exactly one face of $E_{i}$ contains edges of $E_{j}$, we see that $E_{i}$ must have a face bounded by all edges. Thus each $E_{i}$ is either a cycle, an edge, a star or double star (i.e., each edge of a star is doubled; see figure 8).

Now recall that $E_{1}$ and $E_{2}$ have the same number of edges. If one of them is a single edge, so is the other, and this case is trivial. The other situations are studied below using that one can interchange the role of $E_{1}$ and $E_{2}$, and that $G=E_{1} \cup E_{2}$ should come from a knot (and not a link) diagram.

Case 1. Let $q$ be odd. Then the diagram given by construction 2 is a knot diagram iff the one of $G$ (i.e., with $G$ as checkerboard graph) is so.

Case 1.1. Assume first $E_{1}$ is a double star. Note that each vertex of $E_{1}$ must be also a vertex of $E_{2}$, otherwise $G$ gives no knot diagram.

Case 1.1.1. If $E_{2}$ is a double star, then $G$ is a doubled graph, and $D$ is not a knot diagram.

Case 1.1.2. If $E_{2}$ is a star, then $E_{1}$ and $E_{2}$ cannot have the same number of edges (unless $G$ has an isthmus).

Case 1.1.3. Thus $E_{2}$ is a cycle. Using that $E_{1}$ and $E_{2}$ have the same number of edges, and that each vertex of $E_{1}$ is a vertex of $E_{2}$, we see that some edge of $E_{2}$ is parallel to some edge of $E_{1}$. By duality this means that both $E_{1}$ and $E_{2}$ have an isthmus, a contradiction.

Similarly the case is done that $E_{2}$ is a double star.

Case 1.2. Both $E_{1}$ and $E_{2}$ are cycles. They have the same length $n$. Unless $n=2$, no vertex of $E_{i}$ has valence 2 in $G$ (otherwise $E_{j}=E_{i}^{*}$ will have a double edge). Thus $G=E_{1} \cup E_{2}$ is a doubled cycle, which is not self-dual. If $n=2$, we have the 4-crossing diagram $D$.

Case 1.3. Both $E_{1}$ and $E_{2}$ are stars. They have the same number $n$ of leafs (vertices of valence 1 ). If their centers (vertices of valence $>1$ ) are equal, then $G$ is a double star (or has an isthmus), and is not cut-free. If the center of $E_{1}$ is a leaf of $E_{2}$, it is directly checked that we obtain a self-dual graph $G$ with no isthmus only for $n=2$, which gives the 4 crossing diagram. If the center of $E_{1}$ is not a vertex of $E_{2}$, then since $G$ has no isthmus, $G=K_{2, n}$. This graph is not self-dual.

Case 1.4. The final case is that $E_{1}$ is a star and $E_{2}$ a cycle. Again $E_{1}$ and $E_{2}$ have the same number of edges, $E_{2}$ cannot have a vertex of valence 2 in $G$, and $G$ cannot have an isthmus. The only option then is that $G$ is a wheel graph.

Case 2. Now assume $q=2 l$ is even. This goes similarly to case 1 , with the following modifications.

First, $E_{1}$ should have no cycle (or multiple edge). Otherwise, we argued that at least in one of the interior or exterior of that cycle there are no edges of $E_{2}$. When each edge of the cycle is replaced by an even number of crossings in edge direction, we obtain a component of $D$ for this empty interior or exterior, along with some other component(s). Therefore, $D$ is not a knot diagram. With a similar argument, each vertex of $E_{2}$ must be a vertex of $E_{1}$. 
The exclusion of a cycle (or multiple edge) in $E_{1}$ renders obsolete the analoga of cases 1.1 and 1.2. It becomes necessary to mention that the situation here is no longer symmetric in $E_{1}$ and $E_{2}$. Thus we must treat the opposite case to 1.1 , when $E_{2}$ is a double star. This reduces to the opposite of case 1.1.2, which goes as before, with the role of $E_{1}$ and $E_{2}$ swapped (and using that each vertex of $E_{2}$ is a vertex of $E_{1}$ ). Since $E_{1}$ has no cycle, the opposite to case 1.4 (when $E_{1}$ is a cycle, and $E_{2}$ a star) is also unnecessary. Finally, cases 1.3 and 1.4 go through the same way.

Acknowledgment. I would wish to thank to K. Taniyama and R. Shinjo for proposing the problems to me, and to the referee for his helpful comments.

\section{REFERENCES}

[BN] D. BAR-NATAN, On the Vassiliev knot invariants, Topology, 34 (1995), pp. 423-472.

[CP] K. Cvetko-VAh And T. Pisanski, A census of edge-transitive planar tilings, Journal of Combinatorial Mathematics and Combinatorial Computing, 80 (2012), pp. 243-265.

[Cr] P. R. Cromwell, Knots and Links, Cambridge University Press, 2004.

[DL] O. Dasbach And X.-S. Lin, On the Head and the Tail of the Colored Jones Polynomial, Compositio Math., 142:5 (2006), pp. 1332-1342.

[DT] C. H. Dowker And M. B. Thistlethwaite, Classification of knot projections, Topology Appl., 16:1 (1983), pp. 19-31.

[FI] H. Fleischner AND W. ImRICH, Transitive planar graphs, Math. Slovaca, 29:2 (1979), pp. 97-106, http://dml.cz/dmlcz/131785.

[HS] G. HAHN AND G. SABIDUSSI, Graph symmetry: algebraic methods and applications, NATO Science Series C, 1st edition, Springer, 1997, http://books.google.com/ books?id=-tIaXdII8egC.

[HT] J. Hoste And M. Thistlethwaite, KnotScape, a knot polynomial calculation program, available at http://www.math.utk.edu/ morwen.

[Kf] L. H. Kauffman, State models and the Jones polynomial, Topology, 26 (1987), pp. 395-407.

[LT] W. B. R. Lickorish And M. B. Thistlethwaite, Some links with non-trivial polynomials and their crossing numbers, Comment. Math. Helv., 63 (1988), pp. 527-539.

[MT] W. W. Menasco and M. B. Thistlethwaite, The Tait flyping conjecture, Bull. Amer. Math. Soc., 25:2 (1991), pp. 403-412.

[MS] K. Murasugi And A. Stoimenow, The Alexander polynomial of planar even valence graphs, Adv. Appl. Math., 31:2 (2003), pp. 440-462.

[O] M. OzAWA, Closed incompressible surfaces in complements of positive knots, Comment. Math. Helv., 77 (2002), pp. 235-243.

[PV] M. Polyak And O. Viro, Gauss diagram formulas for Vassiliev invariants, Int. Math. Res. Notes, 11 (1994), pp. 445-454.

[Ro] D. Rolfsen, Knots and links, Publish or Perish, 1976.

[St] A. Stoimenow, Positive knots, closed braids, and the Jones polynomial, math/9805078, Ann. Scuola Norm. Sup. Pisa Cl. Sci., 2:2 (2003), pp. 237-285.

[St2] _ Gauß sum invariants, Vassiliev invariants and braiding sequences, Jour. of Knot Theory and its Ramifications, 9:2 (2000), pp. 221-269.

[St3] Coefficients and non-triviality of the Jones polynomial, J. Reine Angew. Math. (Crelle's J.), 657 (2011), pp. 1-55.

[St4] Square numbers, spanning trees and invariants of achiral knots, math.GT/0003172, Comm. Anal. Geom., 13:3 (2005), pp. 591-631.

[St5] Knots of genus one, Proc. Amer. Math. Soc., 129:7 (2001), pp. 2141-2156.

[St6] Knots of (canonical) genus two, math.GT/0303012, Fund. Math., 200:1 (2008), pp. $1-67$.

[St7] _ Some inequalities between knot invariants, Internat. J. Math., 13:4 (2002), pp. 373393.

[St8] Diagram genus, generators and applications, preprint, arXiv:1101.3390.

[St9] On the crossing number of semiadequate links, accepted by Forum Math.

[SA] - (partly with N. Askitas), On Unknotting Numbers and Knot Trivadjacency, Math. Scand., 94:2 (2004), pp. 227-248.

[SV] A. Stoimenow And A. Vdovina, Counting alternating knots by genus, Math. Ann., 333 (2005), pp. 1-27. 
[Th] M. B. Thistlethwaite, A spanning tree expansion for the Jones polynomial, Topology, 26 (1987), pp. 297-309.

[Th2] _ On the Kauffman polynomial of an adequate link, Invent. Math., 93:2 (1988), pp. 285-296.

[Wo] S. Wolfram, Mathematica - a system for doing mathematics by computer, AddisonWesley, 1989. 
